\title{
The mitochondrial genome encodes abundant small noncoding RNAs
}

Seungil Ro ${ }^{1}$, Hsiu-Yen Ma ${ }^{1,2}$, Chanjae Park ${ }^{1}$, Nicole Ortogero ${ }^{1}$, Rui Song ${ }^{1,5}$, Grant W Hennig ${ }^{1}$, Huili Zheng ${ }^{1}$, Yung-Ming Lin $^{2}$, Loredana Moro ${ }^{3}$, Jer-Tsong Hsieh ${ }^{4}$, Wei Yan ${ }^{1}$

${ }^{I}$ Department of Physiology and Cell Biology, University of Nevada School of Medicine, Reno, NV 89557, USA; ${ }^{2}$ Department of Urology, College of Medicine, National Cheng Kung University, 138 Sheng Li Road, Tainan 70403, China; ${ }^{3}$ Institute of Biomembranes and Bioenergetics, National Research Council (CNR), Bari 70123, Italy; ${ }^{4}$ Department of Urology, Southwestern Medical Center, University of Texas, Dallas, TX 75235, USA

Small noncoding RNAs identified thus far are all encoded by the nuclear genome. Here, we report that the murine and human mitochondrial genomes encode thousands of small noncoding RNAs, which are predominantly derived from the sense transcripts of the mitochondrial genes (host genes), and we termed these small RNAs mitochondrial genome-encoded small RNAs (mitosRNAs). DICER inactivation affected, but did not completely abolish mitosRNA production. MitosRNAs appear to be products of currently unidentified mitochondrial ribonucleases. Overexpression of mitosRNAs enhanced expression levels of their host genes in vitro, and dysregulated mitosRNA expression was generally associated with aberrant mitochondrial gene expression in vivo. Our data demonstrate that in addition to 37 known mitochondrial genes, the mammalian mitochondrial genome also encodes abundant mitosRNAs, which may play an important regulatory role in the control of mitochondrial gene expression in the cell.

Keywords: mitochondria; small noncoding RNAs; RNA interference; transcription; gene expression; circular genome Cell Research (2013) 23:759-774. doi:10.1038/cr.2013.37; published online 12 March 2013

\section{Introduction}

Mitochondria are organelles that mainly function as the "powerhouse" by producing ATP through the oxidative phosphorylation system (OXPHOS) in most eukaryotic cells $[1,2]$. Dependent or independent of their energy-producing function, mitochondria are also involved in many other cellular processes, such as cell signaling, cell differentiation, cell death and cell proliferation [3-6]. Mitochondria contain their own genetic materials, i.e., mitochondrial DNA (mtDNA). The mitochondrial genome displays characteristics different from those of its nuclear counterpart, including the use of divergent genetic codes,

\footnotetext{
Correspondence: Wei Yan

Tel: +1-775-784-7765 (Office), +1-775-784-4688 (Lab); Fax: +1-775-7846903

E-mail:wyan@unr.edu

${ }^{5}$ Current address: Department of Molecular and Cell Biology, University of California, Berkeley, 142 Life Sciences Addition \# 3200, Berkeley, CA 94720-3200, USA

Received 18 September 2012; revised 8 January 2013; accepted 28 January 2013; published online 12 March 2013
}

transmission by maternal inheritance, a higher mutation rate, polyploidy status and compact organization, etc. [7]. The transcriptional machinery utilized by mitochondria consists of at least three transcription factors (TFAM and either TFB1M or TFB2M for initiation, and mTERF for termination), mitochondrial RNA polymerase (POLRMT), and mitochondrial poly (A) polymerase, which allows the transcription of a small number of functionally important genes in the mitochondrial genome [8]. In both mice and humans, mtDNA harbors 37 genes encoding 2 rRNA subunits (12S and 16S rRNAs), 22 transfer RNAs (tRNAs) and 13 polypeptides that are all involved in OXPHOS [9]. Mitochondrial defects in these genes have been implicated in a wide variety of degenerative diseases, cardiac dysfunction, aging and cancers [10].

The discovery of numerous small noncoding RNA (sncRNA) species encoded by the nuclear genome, including microRNAs (miRNAs) [11-13], piwi-interacting RNAs (piRNAs) [14-16], endogenous small interfering RNAs (endo-siRNAs)[17-20] and promoter-associated small RNAs (PASRs) [21-23], has changed our view on the regulation of gene expression because we now know 
that these sncRNAs are involved in transcriptional or post-transcriptional regulation, mobile element silencing and chromatin remodeling [18, 24-26]. Here, we report that the mitochondrial genome, like its nuclear counterpart, encodes thousands of small RNAs, which are mainly derived from the sense transcripts of the mitochondrial genes and appear to be involved in the regulation of normal expression of mitochondrial genes.

\section{Results}

The mammalian mitochondrial genome encodes numerous small RNAs

In our efforts of cloning sncRNAs from the mouse ovary using the Sanger sequencing method [27], we identified 12 small RNAs that were uniquely mapped to the mitochondrial genome (Supplementary information, Figure S1A). The expression of the 12 mouse mitochondrial genome-encoded small RNAs (mitosRNAs) and 9 predicted orthologous human mitosRNAs was confirmed using a small RNA RT-PCR method [28] (Supplementary information, Figure S1B and S1C). To define the full mitosRNA transcriptome, we subsequently performed 454 deep sequencing using small RNA libraries of the mouse testis, mouse small intestine and human small intestine. From nearly half a million 454 sequencing reads obtained, we identified 1499 and 2540 non-redundant small RNA sequences (ranging from 12-137nt) that were uniquely mapped to the mouse and human mitochondrial genomes, respectively (Figure 1 and Supplementary information, Tables S2-S5). A total of 1285 murine mitosRNAs and 2110 human mitosRNAs were mapped to the heavy $(\mathrm{H})$ strands of the murine and human mtDNA, respectively. In contrast, 214 mouse mitosRNAs and 430 human mitosRNAs were derived from the light (L) strands of the murine and human mtDNA, respectively. This distribution pattern is consistent with that of other known mitochondrial genes encoding rRNA, tRNA and mRNA (28 on the $\mathrm{H}$ strand and 9 on L strand) (Figure 1 and Table 1). MitosRNAs were mapped to regions throughout the mouse and the human mitochondrial genomes. The majority of mitosRNAs were mapped to known mitochondrial genes (rRNAs, tRNAs and mRNAs) and the D-loop region in a sense orientation, whereas few mitosRNAs were in an antisense orientation (Table 1). Numerous homologous mitosRNAs displayed overlapping sequences in three distinct patterns: 5 '-aligned, staggered and 3 '-aligned (Figure 1 and Supplementary information, Tables S2-S5).

Mapping, nomenclature and structural features of mitos$R N A s$
We grouped mitosRNAs derived from a single mitochondrial gene into a cluster and the host gene is reflected in the name of the cluster (e.g., mmu-mitosR-HR1-1-87 representing all of the 87 mouse mitosRNAs derived from $R n r l$ on the $\mathrm{H}$ strand). A total of 39 mitosRNA clusters were identified, including 37 from known mitochondrial genes, one from the region of origin of replication for $\mathrm{L}$ strand (OL) and one from the D-loop region (Supplementary information, Tables S2-S5). Most of the mitosRNAs were located within a single known mitochondrial gene, whereas few were mapped to a region spanning over two genes (Supplementary information, Table S6). Number of mitosRNAs contained within each of the 39 clusters varies, but the distribution pattern of the number of mitosRNAs among 39 mitosRNA clusters was well conserved between mice (Figure 2A) and humans (Figure 2B). Rnr2, one of the two rRNA genes encoded by the mitochondrial genome, hosts the highest number of mitosRNAs in the two organisms (Figure $2 \mathrm{~A}$ and $2 \mathrm{~B}$ ). Although the majority of mitosRNAs were mapped to their host genes in a sense orientation, a few mitosRNAs were fully complementary to the sense transcript of their host genes, which we termed mitosRNA* to distinguish from their sense counterparts. The major size of mitosRNAs ranged between 30-39 nt in mice and 20-29 nt in humans, although mitosRNAs can be as short as $12 \mathrm{nt}$ and as long as $137 \mathrm{nt}$ in length (Figure 2C). $\mathrm{H}$ strand-derived mitosRNAs usually start with adenine (A) and end in A, cytosine (C), or thymine (T) in both humans and mice, whereas the L strand-derived mitosRNAs appear to prefer "TA" at their 5'-ends and "TT" in the third and fourth positions from the 3 '-termini (Figure 2D).

Differential expression patterns of mitosRNAs among organs and species

We then analyzed the expression of five mouse mitosRNA clusters and their human orthologs in multiple organs (Figure 3A) using a PCR-based small RNA quantification method [28]. One mitosRNA cluster contains all of the homologous mitosRNAs displaying overlapping sequences with small differences in either their 5'ends, middle portions or $3^{\prime}$-ends in three distinct patterns: 5'-aligned, staggered and 3'-aligned (Supplementary information, Tables S2-S5), which can be amplified simultaneously by the PCR method [28]. PCR products contain amplicons with a size ranging between $\sim 100-120 \mathrm{bp}$, including sequences of the mitosRNA ( 20-40 nt) and the miRTQ linker (84 nt) [28]. Therefore, the PCR products appeared as thicker bands in gel images (Figure 3A). The amplicons were sequenced and confirmed to contain all of the homologous mitosRNAs (Supplementary informa- 
A
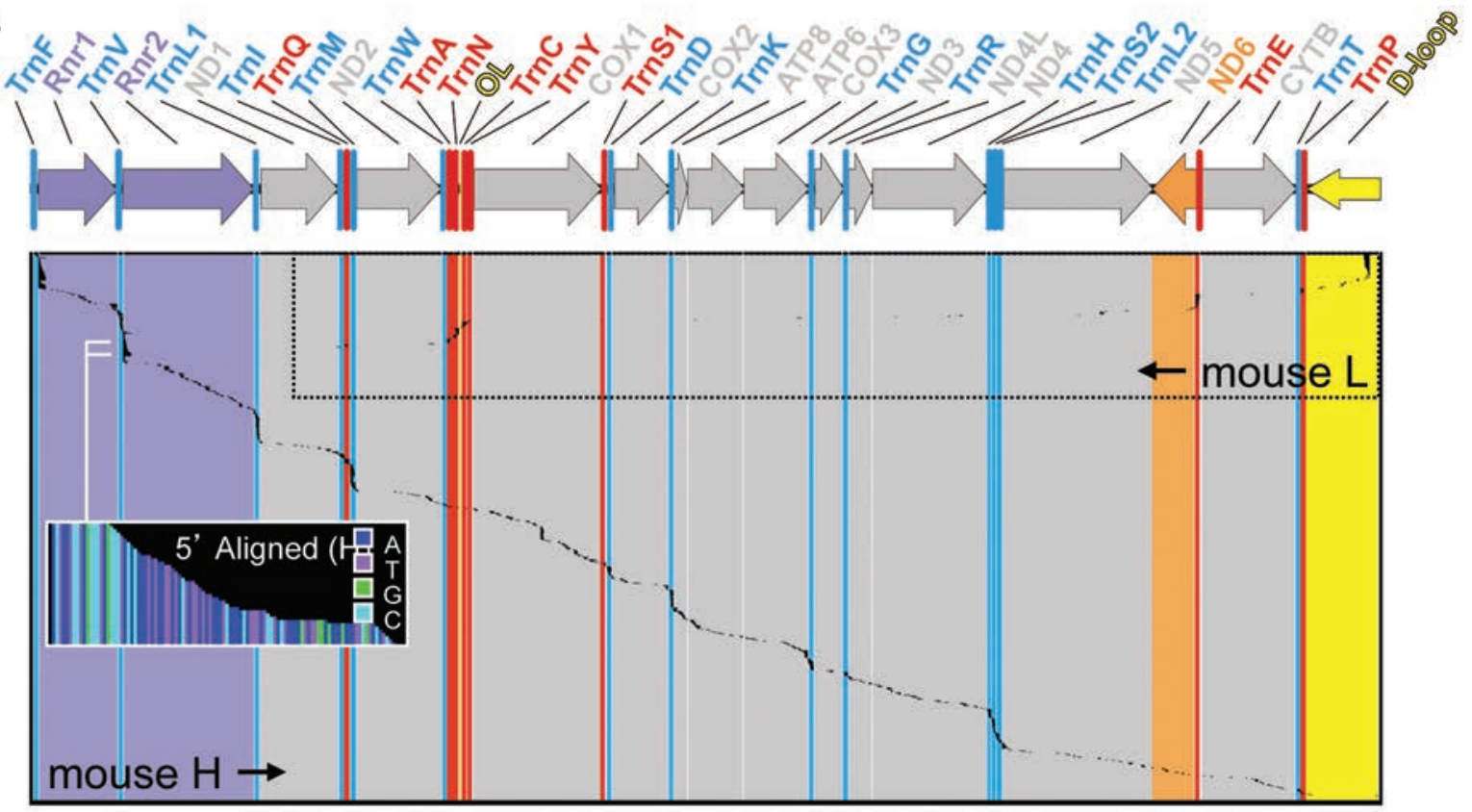

B

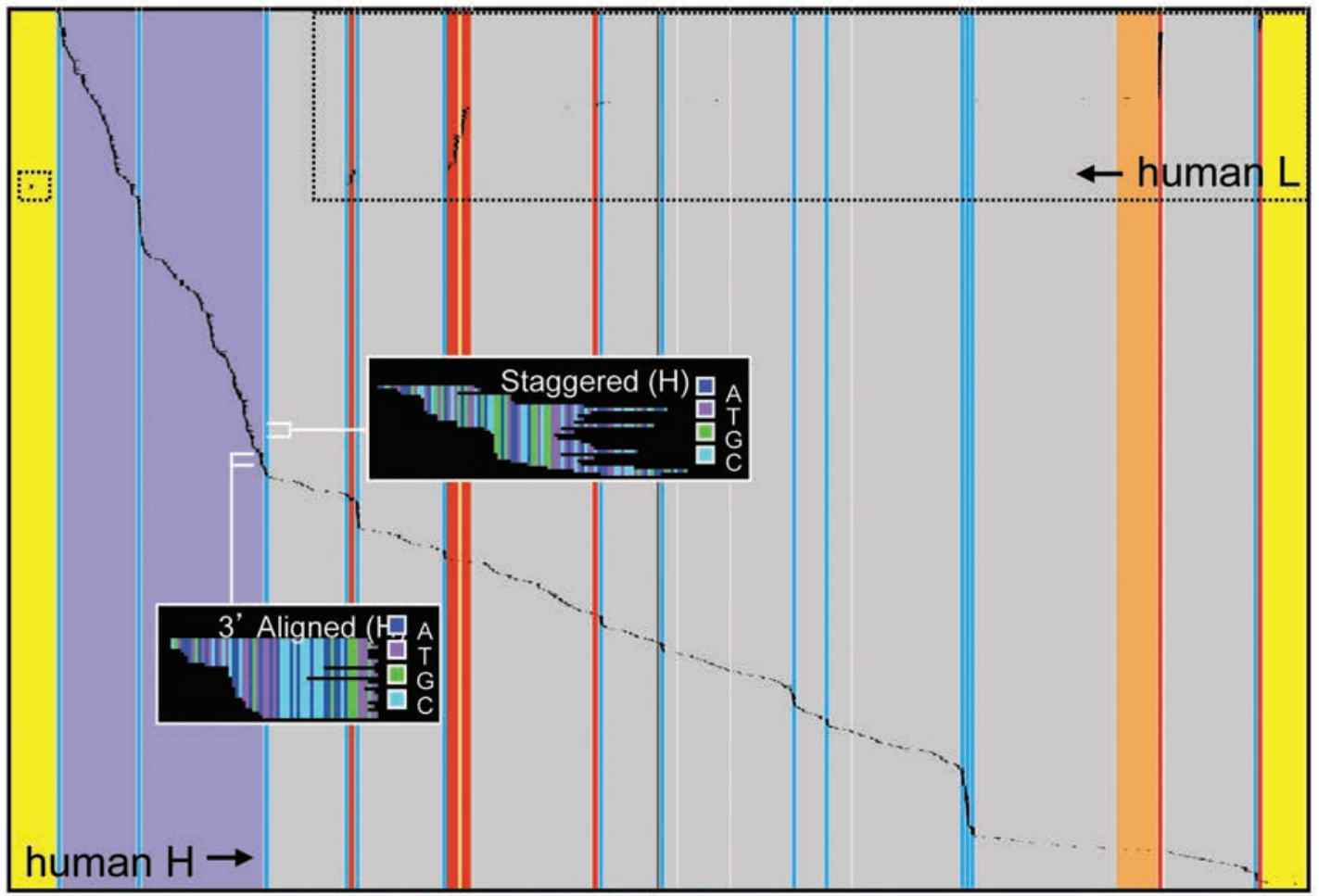

Figure 1 Mapping of mitosRNA sequence reads to the mitochondrial genome. (A) 1499 non-redundant mouse mitosRNAs were mapped to the mouse mitochondrial genome. 1285 and 214 mouse mitosRNAs were mapped to the $H$ and $L$ strands, respectively. (B) 2540 human mitosRNAs were mapped to the human mitochondrial genome. 2110 and 430 human mitosRNAs were mapped to the $\mathrm{H}$ and $\mathrm{L}$ strands, respectively. Note that the majority of mitosRNAs were mapped to known mitochondrial genes (rRNA, tRNA and mRNA) and the D-loop region in a sense orientation. Representative alignment patterns of mitosRNA homologs, including 5'-aligned, staggered and 3'-aligned, are shown as insets. The 37 mitochondrial genes are indicated on the map in different colors: blue, tRNAs on $\mathrm{H}$; purple, rRNAs on $\mathrm{H}$; gray, mRNAs on $\mathrm{H}$; red, tRNAs on $\mathrm{L}$; orange, mRNA on $L$. Origin of replication for $L(O L)$ and $D$-loops are also indicated in yellow. 
Table 1 Mapping of mitosRNAs to mouse and human mitochondrial genomes*

\begin{tabular}{|c|c|c|c|c|c|c|c|c|}
\hline Organism & Strand & Orientation & Total mitosRNAs & tRNAs (22) & rRNAs (2) & mRNAs (13) & D-loop & $\mathbf{O L}^{2}$ \\
\hline \multirow[t]{3}{*}{ Mouse } & $\mathrm{H}(85 \%)$ & Sense & $857(96 \%)$ & $277(14)$ & $218(2)$ & $362(12)$ & 0 & 0 \\
\hline & & Antisense & $39(4 \%)$ & 23 & 0 & 13 & 3 & 0 \\
\hline & & Antisense & $21(14 \%)$ & 2 & 1 & 18 & 0 & 0 \\
\hline \multirow[t]{2}{*}{ Human } & $\mathrm{H}(83 \%)$ & Sense & $1481(99 \%)$ & $390(14)$ & $684(2)$ & 407 (12) & 0 & 0 \\
\hline & & Antisense & $13(4 \%)$ & 3 & 0 & 10 & 0 & 0 \\
\hline
\end{tabular}

${ }^{*} \mathrm{H}$ and L strands encode 28 and 9 genes, respectively. Most mitosRNAs are mapped on H strands in a sense orientation.

${ }^{1}$ Numbers of genes

${ }^{2}$ Origin of replication for $\mathrm{L}$ strand

tion, Tables S2-S5). The five mouse mitosRNA clusters were detected in all of the seven organs analyzed and the five human mitosRNA clusters appeared to display organ specificity (e.g., hsa-mitosR-H-Q*_1-5 and H-X1_38-42 in Figure 3A). Sequencing analyses of the PCR products revealed that mitosRNA contents in each of the clusters were similar in the mouse organs, but were different in the human brain and heart vs. gastrointestinal tissues (e.g., for hsa-mitosR-H-R2_1-16, longer mitosRNAs were detected in the brain and heart, whereas shorter mitosRNAs were detected in the gastrointestinal tissues) (Figure 3A, right upper panel). Similar phenomena were observed in expression patterns of 12 mouse mitosRNAs cloned from the ovary and 9 predicted orthologous human mitosRNAs (Supplementary information, Figure S1B and S1C), suggesting that expression of mitosRNAs may be more tissue-specific in humans than in mice.

MitosRNAs are not RNA turnover products, but generated by ribonucleases

Small RNAs, including miRNAs and small nucleolar RNAs (snoRNAs), which are generated by ribonucleases, contain 5' phosphate and 3' hydroxyl termini, whereas most RNA turnover products possess a $5^{\prime}$ hydroxyl terminus [29]. To confirm that these mitosRNAs were not RNA turnover products, we next examined whether mitosRNAs contained a phosphate group at their $5^{\prime}$ ends. Small RNA cDNA libraries of mouse or human small intestine were constructed with or without dephosphorylation of small RNAs (Figure 3B). Five mouse (Figure 3C, left panel) and 5 human (Figure 3C, right panel) mitosRNAs were amplified by PCR using each mitosRNA-specific primer and a universal primer miP2 (Supplementary information, Table S1). No amplification was observed in any of the 5 murine or 5 human mitosRNAs when dephosphorylated small RNAs were used to construct the small RNA cDNA library (Figure $3 \mathrm{C}$, bottom panels), whereas all were amplified from the non-dephosphorylated small RNA cDNA libraries (Figure $3 \mathrm{C}$, top panels). The same result was obtained from two controls, including a miRNA let-7d and a snoRNA U80 (Figure 3C). Sequencing analyses of the PCR products confirmed that all five mouse mitosRNAs were indeed amplified (Supplementary information, Tables S2-S5). Among five human mitosRNAs, two showed perfect match to the cloned mitosRNAs (Supplementary information, Table S3) except hsa-mitosR-H-Q*_1-5 (missing $2 \mathrm{nt}$ at $5^{\prime}$ ), hsa-mitosR-H-X1 38-42 (containing an extra 23 nt at $5^{\prime}$ ) and hsa-mitosR-L-DL_4-10 (containing 3-5 nt at $5^{\prime}$ ). The three unmatched human mitosRNAs were those predicted based on their mouse orthologs. Sequencing analyses confirmed that PCR products of the two control small RNAs let-7d and U80 all matched to their sequences. The $\sim 230$ bp PCR products amplified along with the mitosRNAs represented the full-length cDNA generated using the primer pair miP2 and RTQUN1r during PCRs (Figure 3B). These data indicate that mitosRNAs have a phosphate group at their $5^{\prime}$-ends, as do miRNAs and snoRNAs, suggesting that they represent small RNA products generated by yet-to-be-identified ribonucleases.

\section{MitosRNAs are localized to mitochondria}

We then purified mitochondria from murine brain or liver cells, and performed northern blot analyses using small RNAs isolated from mitochondrial and cytosolic fractions (Figure 4A-4F). Mmu-mitosR-L-A 3 (derived from TrnA on L strand) and mmu-mitosR-L-P+ 6 (from TrnP on L strand) were detected along with TrnA and TrnP in mitochondrial fractions, but not in cytosolic fractions of brain cells (Figure 4A and 4B). Mmu-mitosR-H$A^{*}$ _ (antisense to TrnA on $\mathrm{H}$ strand) and mmu-mitosR- 
A

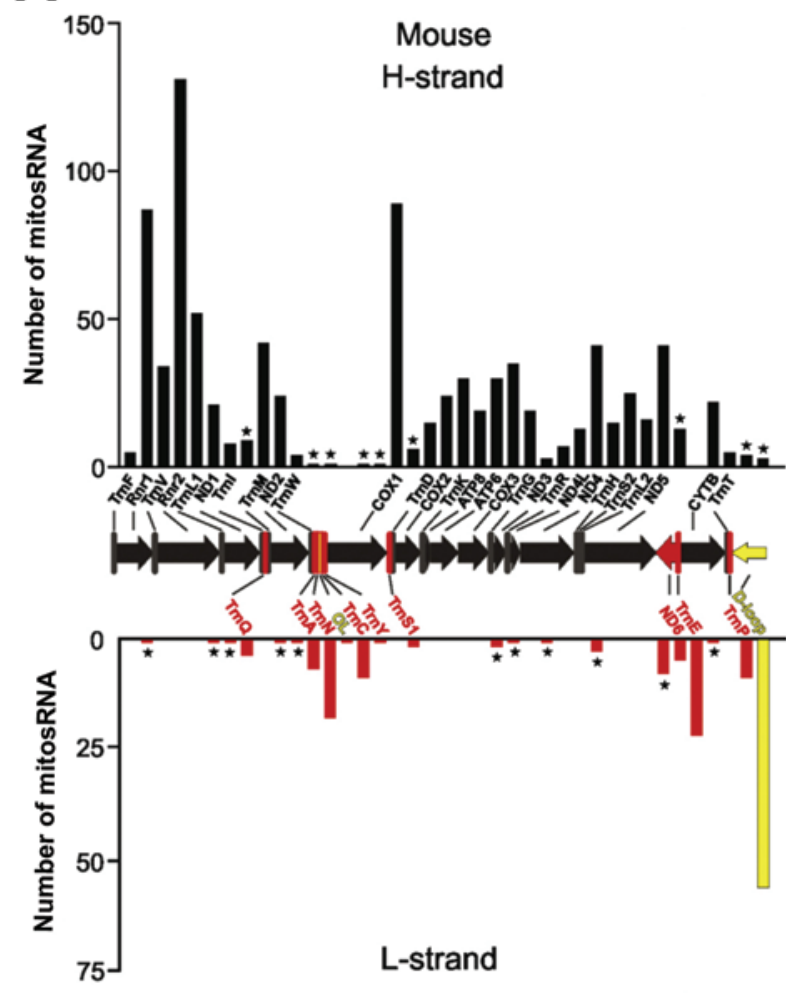

C

C

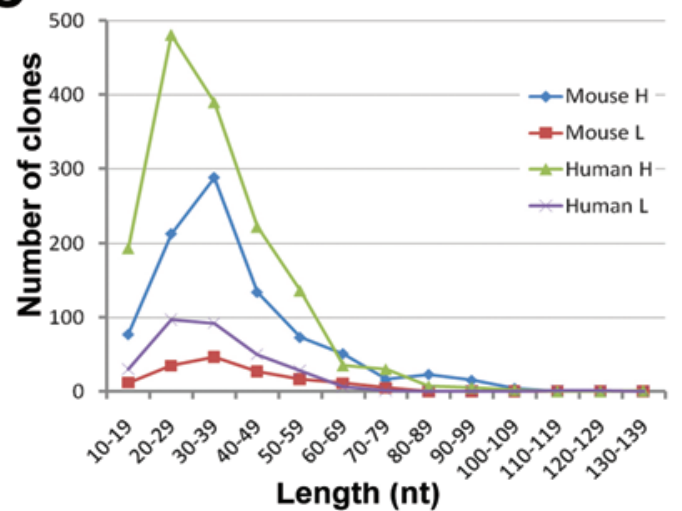

B

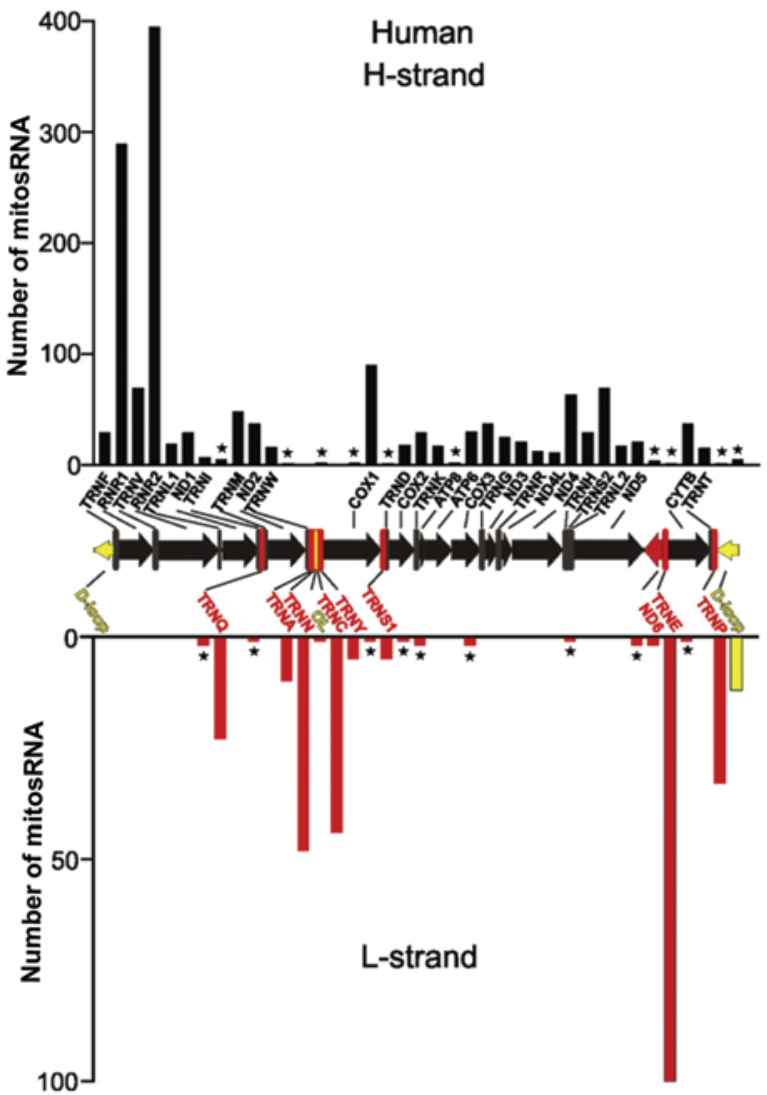

D
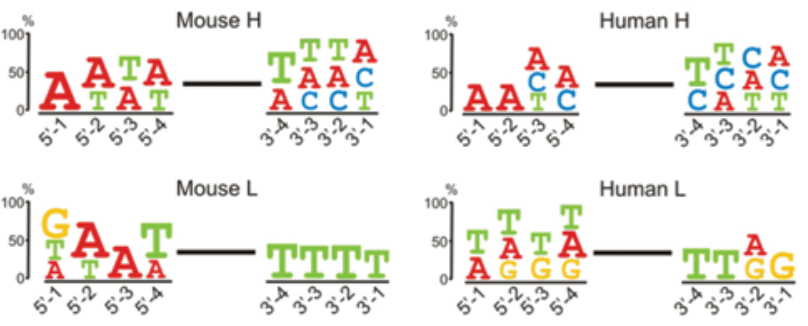

Figure 2 MitosRNA clusters, size distribution and 5'- or 3'-end sequence motifs. (A-B) Number of mitosRNAs in each of the 39 mitosRNA clusters identified in mice (A) and humans (B). MitosRNAs derived from a specific mitochondrial gene, origin of replication for $L(O L)$ or $D$-loop were grouped into one cluster. Bars represent number of mitosRNAs mapped to the heavy $(H)$ strand (in black), to the light (L) strand (in red) and to the D-loop and OL (in yellow) of the mitochondrial genome. A star ( $\star$ ) indicates mitosRNAs mapped to the antisense transcripts. (C) Size distribution of mitosRNAs derived from $\mathrm{H}$ or $\mathrm{L}$ strand of mouse and human mtDNA. The most common sizes are 30-40 nt for mouse mitosRNAs and 20-30 nt for human mitosRNAs. (D) Nucleotide preference at the 5'- and 3'-ends of mitosRNAs.

H-P*__3 (antisense to TrnP on H strand) were detected in all six organs analyzed, with the highest levels in heart (Figure 4C and 4D). These two antisense mitosRNAs were also exclusively detected in mitochondria of both brain and liver cells (Figure 4E and 4F). Taken together, mitosRNAs appear to be exclusively localized to mito- 
A

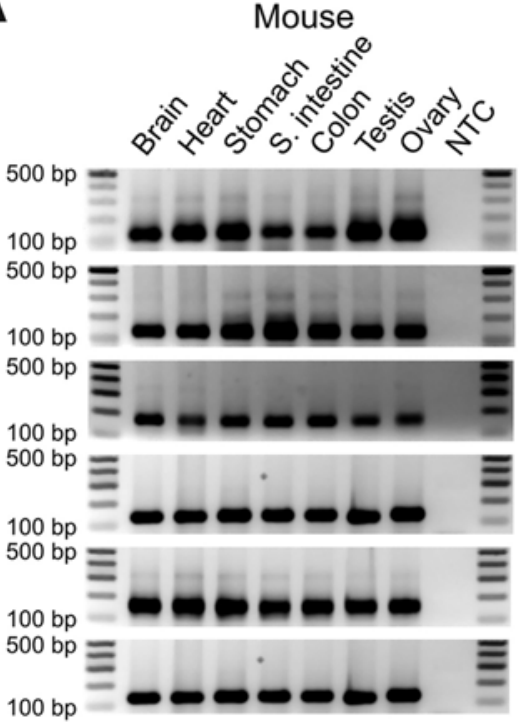

Human

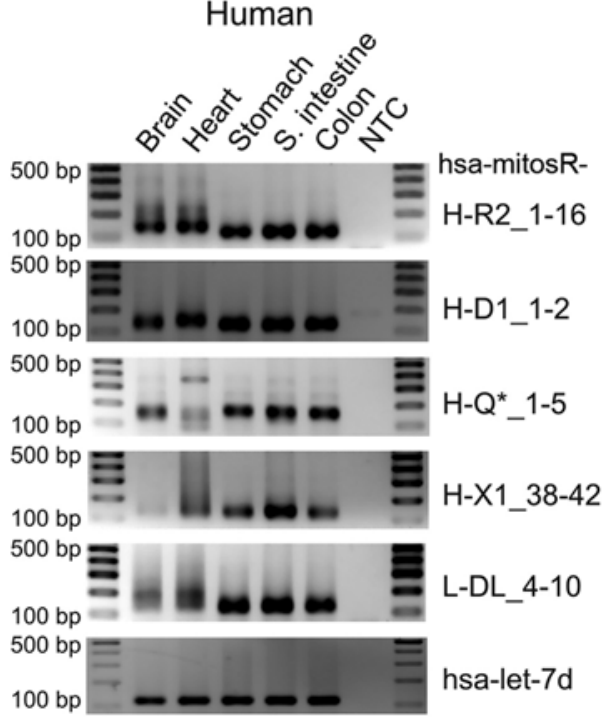

B
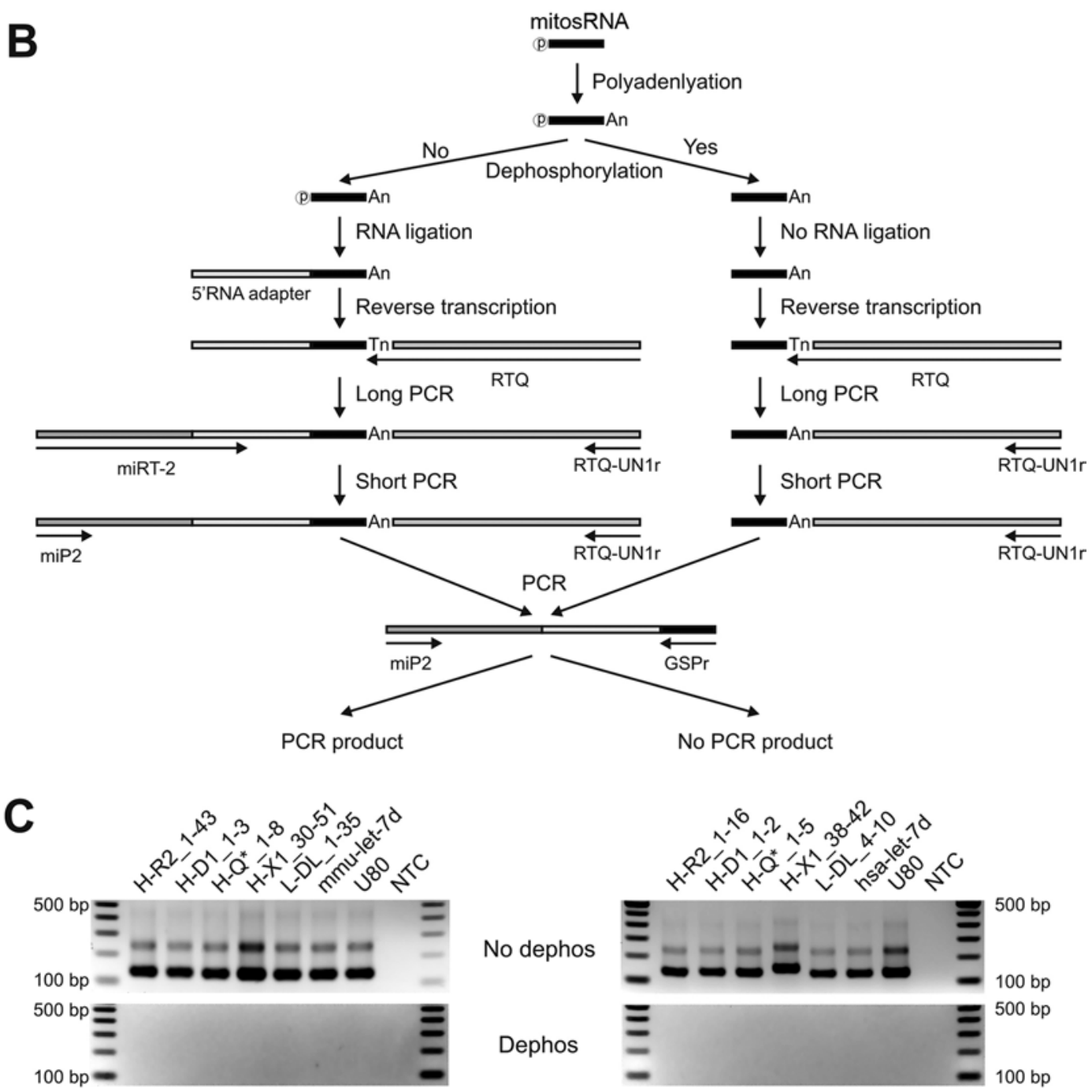
chondria.

MitosRNAs are indeed encoded by the mitochondrial genome rather than nuclear mtDNA-derived pseudogenes

To exclude the possibility that mitosRNAs are derived from transcripts of numerous nuclear pseudogenes of mitochondrial DNA [30-32], we performed mitosRNA deep sequencing (mitosRNA-Seq) using wild-type (WT) and mtDNA-depleted (Rho0) cells of PNT1A (an immortalized human prostate epithelial cell line) cells [33]. Compared with WT PNT1A cells, levels of all mitosRNAs were drastically reduced or largely absent in Rho0 PNT1A cells (Figure 5 and Supplementary information, Table S7). The global reduction in mitosRNA transcriptome correlated well with the global depletion of mitochondrial DNA, suggesting that mitosRNAs are truly encoded by the mitochondrial genome.

MitosRNAs target antisense transcripts and enhance the expression of their host mitochondrial genes in vitro

Mmu-mitosR-L-A_3 and mmu-mitosR-L-P+_6 are mapped to the 3 '-half of $\operatorname{Trn} \mathrm{A}$ and $\mathrm{TrnP}$ in a sense orientation, respectively (Figure 6A and 6B). To test the effects of these two mitosRNAs on the expression of their host genes TrnA and TrnP, we transfected the NIH 3T3 cells with L-A_3-sen (sense), L-P+ 6-sen (sense), L-A_3-anti (antisense) or L-P+_6-anti (antisense) RNA oligos. Non-specific scrambled RNA oligos, mismatched RNA oligos and samples without transfection were used as controls. Expression levels of TrnA and TrnP were almost doubled when L-A_3-sen and L-P+_6-sen was introduced to the cells, respectively (Figure $\overline{6 A}$ and $6 \mathrm{~B}$ ). In contrast, TrnA and TrnP levels were significantly reduced when cells were transfected with L-A_3-anti and $\mathrm{L}-\mathrm{P}+6$-anti oligos, respectively (Figure $6 \mathrm{~A}$ and $6 \mathrm{~B}$ ). No effects were observed when scrambled or irrelevant/ mismatched small RNA oligos were used in transfection (Figure 6A and 6B). A similar experiment was performed to examine the effects of mmu-mitosR-H-X1_46 on its target, mitochondrial Cox 1 mRNAs. Mmu-mitosR-HX1_46 was mapped to the middle of the Cox 1 open read- ing frame (ORF). Expression levels of Coxl mRNA were dramatically reduced with $\mathrm{H}-\mathrm{X} 1$ 4 46-anti oligos, whereas its levels were increased with H-X1_46-sen oligos (Figure $6 \mathrm{C}$ and $6 \mathrm{D}$ ). These results suggest that mitosRNAs can enhance the production of their host mitochondrial genes, whereas anti-mitosRNAs can inhibit the expression of their host mitochondrial genes in vitro.

DICER inactivation correlates with aberrant mitosRNA production and dysregulated expression of mitochondrial genes

Ribonuclease III DICER can cleave double-stranded RNAs (dsRNAs) and RNAs with a stem-loop structure into endo-siRNAs and miRNAs, respectively [18]. To explore whether mitosRNAs are direct products of DICER, we examined mitosRNA levels in Dicer-null pachytene spermatocytes (one of the male germ cell types) $[17,34]$ and Dicer-null Sertoli cells (one of the testicular somatic cell types) [35] using a small RNA qPCR method [28] (Figure 7). These Dicer-null germ and somatic cell types were purified from a male germline-specific Dicer conditional knockout (cKO) mouse line $[17,34]$ and a Sertoli cell-specific Dicer cKO line [35] generated previously. Expression of eGFP in Cre-expressing cells in these $\mathrm{cKO}$ lines allowed us to purify Dicer-null pachytene spermatocytes and Sertoli cells with the purity of $>95 \%[17$, 34].

Levels of nine mitosRNA clusters examined were decreased by $\sim 20-90 \%$ in Dicer-null pachytene spermatocytes compared with levels of these mitosRNA clusters in controls (Figure 7A). In Sertoli cells, one out of nine mitosRNA clusters analyzed showed upregulated levels, whereas levels of two remained unchanged and six displayed significantly reduced levels (Figure 7E). Given that Dicer inactivation cannot abolish mitosRNA production, it is highly unlikely that mitosRNAs are direct products of Dicer cleavage, and the altered mitosRNA expression may reflect secondary effects of Dicer inactivation in these cells.

Consistent with the aberrant expression levels of mitosRNAs in Dicer-null pachytene spermatocytes and

Figure 3 Validation of expression and the 5' phosphate group of cloned mitosRNAs. (A) Expression of 5 murine mitosRNA clusters (left) and 5 human mitosRNA clusters (right) in multiple organs was detected using a small RNA RT-PCR method [28]. All PCR products were gel-eluted and sequenced to confirm the sequence identity (Supplementary information, Tables S2-S5). miRNA let-7d was used as a loading control. (B) A schematic diagram showing construction of the mitosRNA cDNA libraries with or without dephosphorylation followed by amplification of the $5^{\prime}$-ends of the libraries. Note that the $5^{\prime}$ RNA linker cannot be ligated to small RNAs without the $5^{\prime}$ phosphate and thus cannot be amplified by PCR. (C) Confirmation of the existence of $5^{\prime}$ phosphate on the identified mitosRNAs. MitosRNA cDNA libraries of mice (left) and humans (right) were constructed with or without dephosphorylation followed by PCR amplification. A housekeeping miRNA (let-7d) and a housekeeping snoRNA (U80) were used as loading controls. All PCR products in A and B were subcloned into a TA cloning vector and subjected to sequencing. 

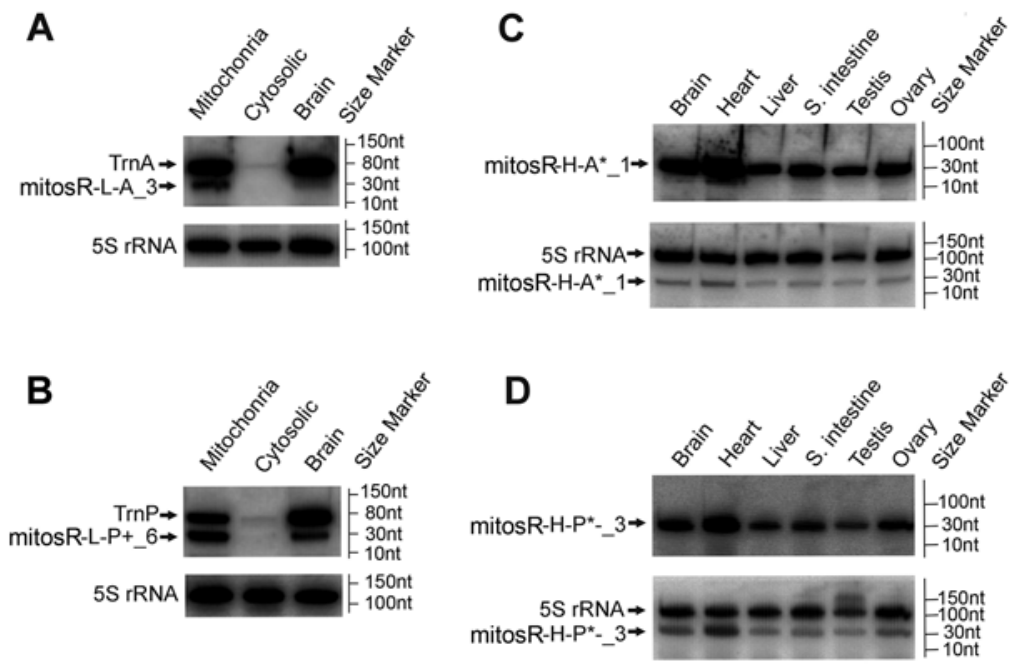
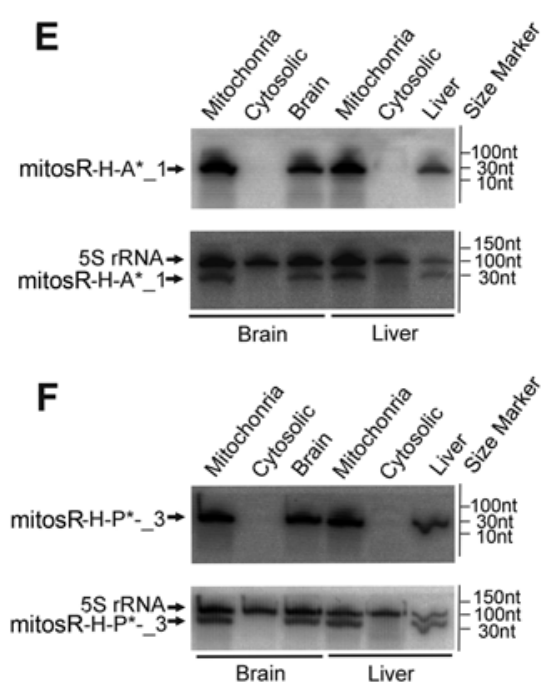

Figure 4 Subcellular localization of mitosRNAs. (A-B) Northern blot analyses of TrnA and mitosR-L-A_3 (A) or TrnP and mitosR-L-P+_6 (B) in mitochondrial and cytosolic fractions of mouse brain cells. (C-D) Detection of mitosR-H-A ${ }^{*}{ }^{1}$ (antisense of TrnA) (C) or mitosR-H-P*_3 (antisense of TrnP) (D) in 6 mouse organs using northern blot analyses. (E-F) Northern blot analyses of mitosR-H-A*_1 (E) or mitosR-H-P*_-3 (F) in mitochondrial and cytosolic factions of mouse brain or liver cells. Note that each membrane was partially stripped and re-probed for $5 \mathrm{~S}$ rRNA, which was used as a loading control.

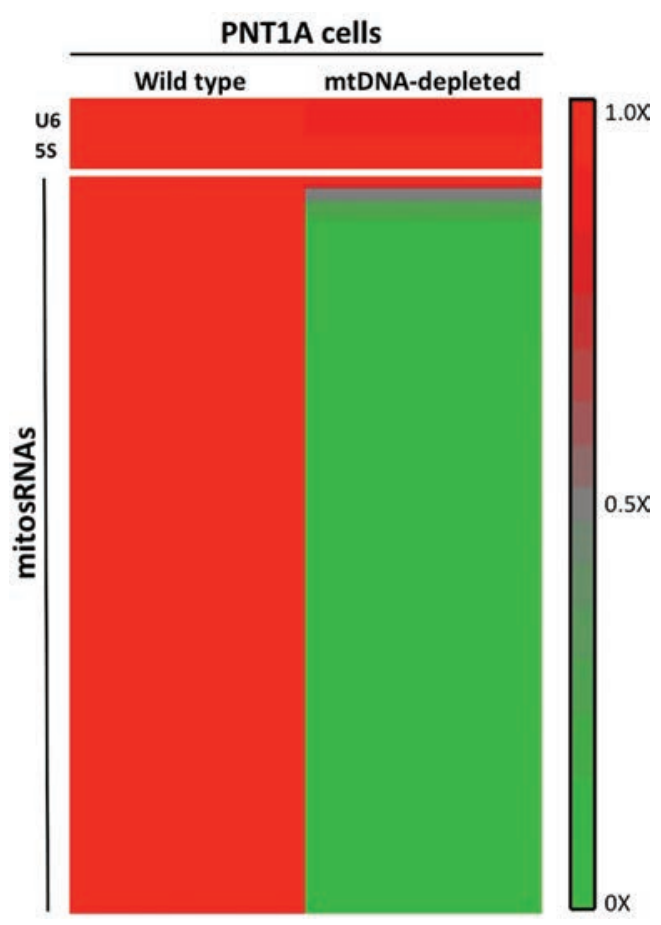

Figure 5 Heat map showing expression levels of mitosRNAs in WT and mtDNA-depleted (Rho0) PNT1A cells, as determined by mitosRNA deep sequencing analyses (mitosRNA-Seq). Levels of mitosRNAs are presented as values relative to those in wildtype cells, i.e., $1 \times$ indicates levels greater than or equal to those in WT cells and $0.5 \times$ represents an expression level that is half of what was observed in WT cells (see original data in Supplementary information, Table S7).
Sertoli cells (Figure 7A and 7E), levels of mitochondrial rRNAs (Figure 7B and 7F), mRNAs (Figure 7C and $7 \mathrm{G}$ ) and tRNAs (Figure 7D and 7H) in these cell types appeared to be dysregulated as well. Levels of two mitochondrial rRNAs (Figure 7B) and 13 mitochondrial mRNAs (Figure 7C) were unchanged or slightly downregulated in Dicer-null pachytene spermatocytes, whereas a $\sim 5$-fold decrease in the levels of the same two rRNAs (Figure 7F) and 13 mRNAs (Figure 7G) was observed in Dicer-null Sertoli cells. Nine of the 22 mitochondrial genome-encoded tRNAs were analyzed and all were downregulated in both Dicer-null pachytene spermatocytes (Figure 7D) and Dicer-null Sertoli cells (Figure $7 \mathrm{H})$. These results suggest that Dicer inactivation can cause aberrant mitosRNA production and dysregulated expression of mitochondrial genes. However, the causeeffect relation between the aberrant mitochondrial gene expression and dysregulated mitosRNA production in Dicer-null cells remains unclear.

No known components of the RNAi machinery are present inside mitochondria

The findings that mitosRNAs can enhance expression levels of their host mitochondrial genes in vitro and aberrant mitosRNA expression correlates with disrupted expression of mitochondrial tRNA, mRNA or rRNA genes in vivo suggest that mitosRNAs may function to regulate mitochondrial gene expression. One of the most likely mechanisms would be the RNA interference 
A
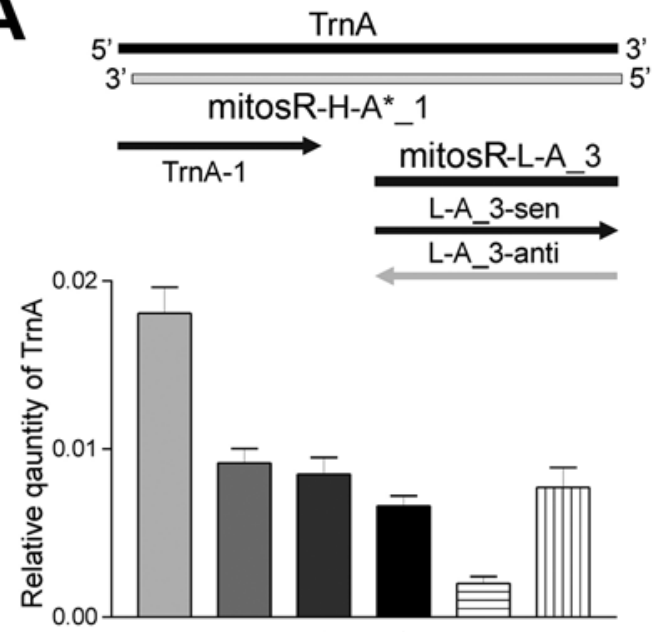

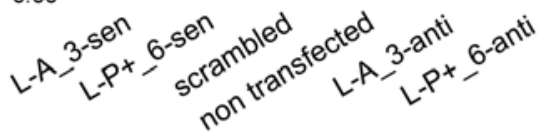

C

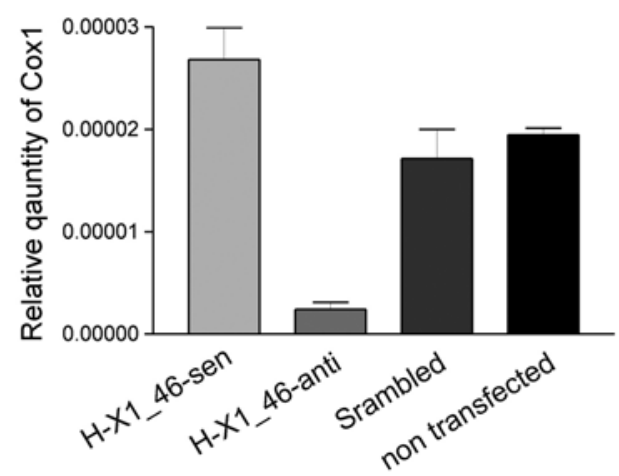

B
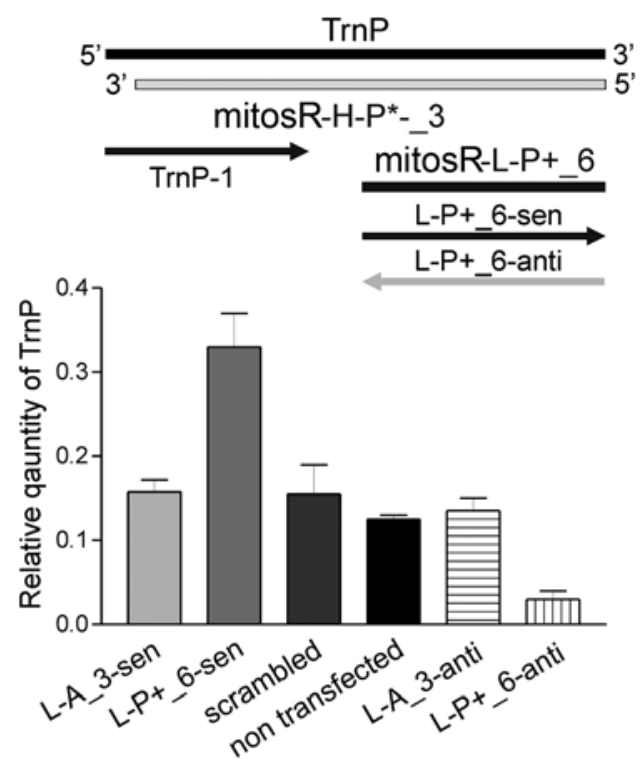

D

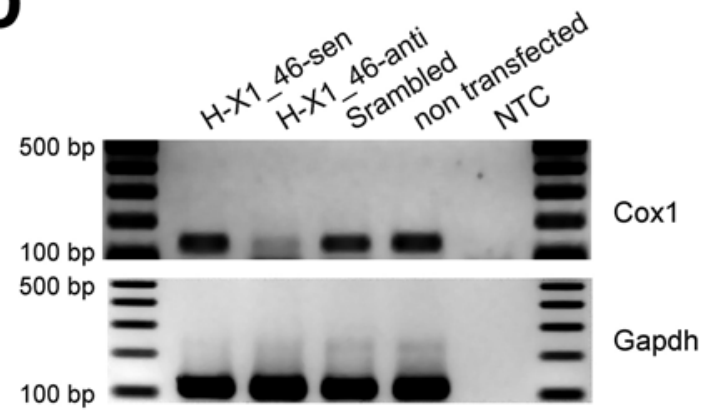

Figure 6 Effects of mitosRNAs on mitochondrial gene expression in vitro. (A-D) Effects of sense or antisense mitosRNAs on levels of their host/target tRNAs (A and B) and Cox1 (C and D) in NIH 3T3 cells. In the very top two panels, solid lines represent tRNA genes, whereas gray lines underneath represent antisense transcripts of the tRNAs (mitosR-H-A* 1 and mitosRH-P*-_3). Sense and antisense mitosRNAs (e.g., L-A_3-sen and L-A_3-anti for mitosR-L-A_3) were indicated by arrows. Primers (e.g., TrnA-1 for TrnA) used for qPCR were also indicated by arrows. SYBR green-based qPCR was performed to determine levels of tRNAs and let-7d was used as an internal loading control for normalization (A and B). qPCR was also performed on Cox1 mRNAs and Gapdh was used for normalization (C and D). Negative controls included nonspecific scrambled mitosRNAs, non-targeting mitosRNA (e.g., transfections with L-P+_6-sen or L-P+_6-anti followed by examining levels of TrnA, which is not their host/target mitochondrial gene) and non-transfected cells.

(RNAi) mechanism, which is utilized by miRNAs and siRNAs to control mRNA stability and translational efficiency in eukaryotic cells [36]. To examine whether components of the RNAi machinery are present inside the mitochondria, we purified mitochondria from human HEK293T cells and examined the expression of DICER and AGO2 (Supplementary information, Figure S2AS2C). TOMM22 and COX4, two mitochondrial marker proteins, were predominantly detected in the purified mi- tochondrial fractions, in which GAPDH and $\beta$-Tubulin, two cytosolic proteins, were absent, suggesting that the isolated mitochondria were highly enriched with negligible cytoplasmic contaminations (Supplementary information, Figure S2A-S2B). Although Dicer and AGO2 were readily detected in the total cell lysates, these two proteins were absent in highly enriched mitochondrial fractions, suggesting that mitochondria do not contain the known RNAi components. 

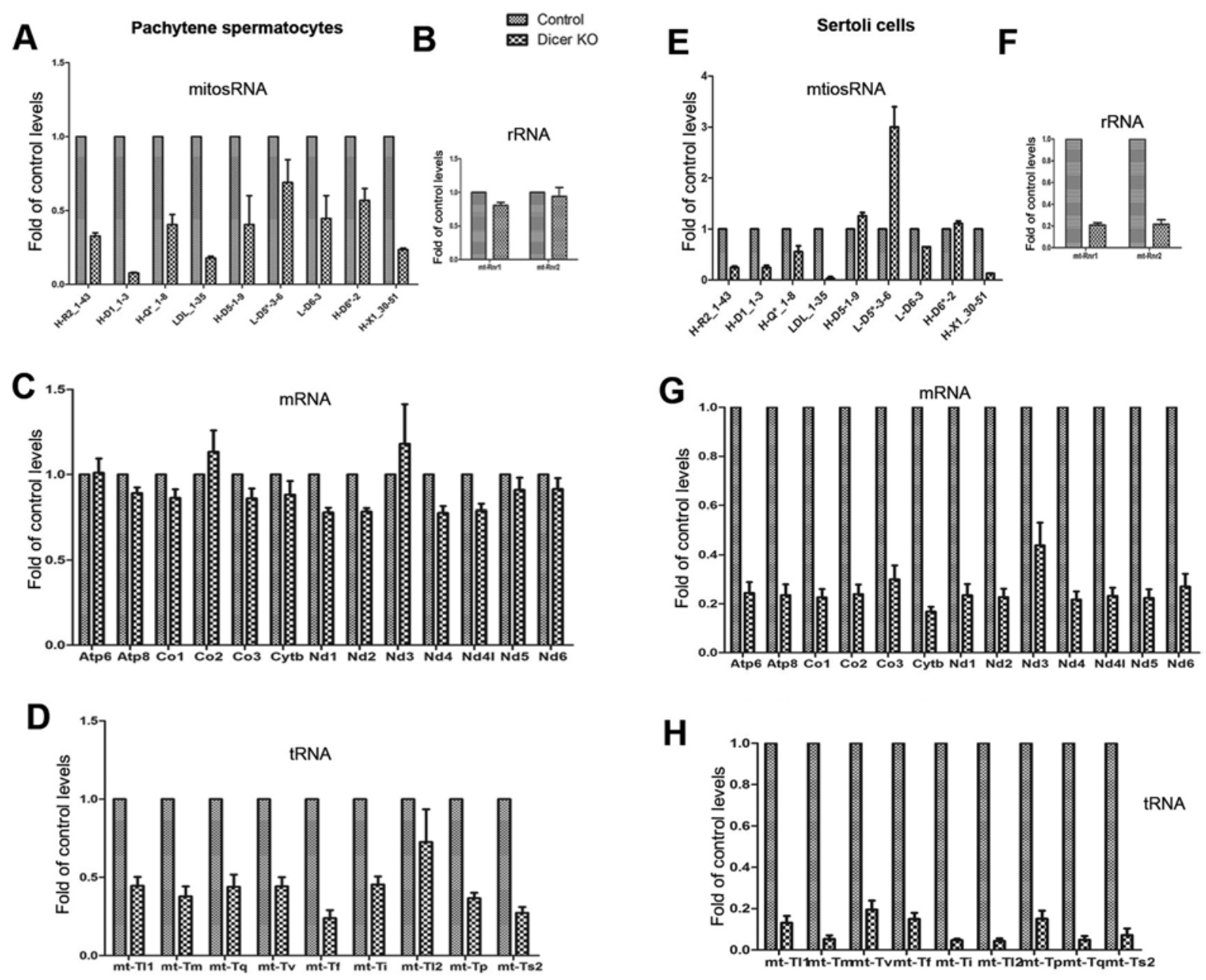

Figure 7 Aberrant expression of mitosRNAs and mitochondrial genes in control and Dicer-null pachytene spermatocytes or Sertoli cells. (A-D) qPCR analyses of levels of 9 mitosRNA clusters (A), two mitochondrial rRNAs (B), 13 mitochondrial mRNAs (C) and 9 mitochondrial tRNAs (D) in pachytene spermatocytes purified from control ( $\mathrm{mTmG}^{+/ t g}$; Stra8-iCre) and Dicer cKO (Dicer ${ }^{\text {dox/ }} ; m T m G^{\text {thtg }}$; Stra8-iCre) mice. (E-H) qPCR analyses of levels of 9 mitosRNA clusters (E), two mitochondrial rRNAs (F), 13 mitochondrial genome-encoded mRNAs (G) and 9 mitochondrial tRNAs (H) in Sertoli cells purified from control $\left(m T \mathrm{FG}^{+/ t g} ; \mathrm{Amh}-\mathrm{Cre}\right)$ and Dicer cKO (Dicer $\left.{ }^{\text {lox/lox }} ; \mathrm{mTmG}^{+/ t g} ; \mathrm{Amh}-\mathrm{Cre}\right)$ mice. Note that qPCR analyses were performed in triplicates and each cell type was purified using pooled testes from multiple mice. Relative levels on the $y$-axis represent ratios of levels in Dicer-null cells/levels in control cells.

We further searched several known mammalian mitochondria proteomic databases [37-39] and failed to find any of the known components of the RNAi machinery (e.g., DICER, AGO2, MVH, etc.). These results are consistent with the fact that mitosRNAs are not products of DICER cleavage. Therefore, the biogenesis of mitosRNAs must be mediated by one or more currently unidentified mitochondrial ribonucleases, which may be under the regulation of DICER or DICER-dependent sncRNAs. The lack of any known components of the RNAi machinery inside mitochondria suggests that either mitosRNAs do not function at post-transcriptional levels or there is an alternative RNAi-like machinery operating inside mitochondria.

\section{Discussion}

The mammalian mitochondrial genome is a large circular double-stranded DNA, which contains a total of 37 genes encoding 2 rRNAs, 22 tRNAs and 13 mRNAs [6]. The majority of mitochondrial genes are located on the heavy $(\mathrm{H})$ strand (14 tRNA, 2 rRNA and 12 mRNA genes), whereas the light (L) strand harbors 8 tRNA and 1 mRNA genes in both mice and humans. Both strands 
$(\mathrm{H}$ and $\mathrm{L})$ are transcribed from their own promoters: $\mathrm{H}$ strand promoter (HSP) or L strand promoter (LSP) located in the D-loop region [1]. From each promoter, a long polycistronic transcript is generated by a mitochondrial RNA polymerase (Polrmt) [1, 2, 7]. Given that mitochondria possess their completely independent transcription machinery, it is not surprising that the mitochondrial genome, like its nuclear counterpart, also encodes small regulatory RNAs, which we report here as mitosRNAs. Several lines of evidence suggest that mitosRNAs are not random degradation products of mitochondrial transcripts, but rather represent small RNAs processed by unknown ribonuclease(s). First, the size of majority ( $>90 \%$ ) of the murine and human mitosRNAs ranges from $20 \mathrm{nt}$ to 40 nt and their $5^{\prime}$ - or 3'-ends display nucleotide preference (Figure 2C, 2D and Supplementary information, Tables S2-S5). Random distribution of RNA sizes and no nucleotide preference in either end would have been observed if they were RNA turnover products. Second, 86\%-96\% of murine mitosRNAs and $96 \%-99 \%$ of human mitosRNAs are in the sense orientation (Table 1), suggesting a selective mechanism during the biogenesis of mitosRNAs. Third, mitosRNAs all contain a 5'-phosphate terminus (Figure 3), whereas RNA random degradation products usually possess a 5'-hydroxyl terminus [29]. Fourth, distinct bands with expected sizes were readily detected in northern blots (Figure 4), and sequencing analyses confirmed that the PCR products indeed contained the sequences expected (Supplementary information, Tables S2-S5). Finally, mitosRNAs can modulate the expression of their host mitochondrial genes in vitro and dysregulated mitosRNAs correlate with aberrant expression of mitochondrial genes in vivo. All of the evidence suggests that mitosRNAs are synthesized by the cell and may play a pivotal role in the control of normal mitochondrial gene expression.

Bidirectional transcription of the mitochondrial genome inevitably generates the $\mathrm{H}$ - and L-strand transcripts that are complementary to each other. If the antisense transcript was given a chance to bind to the sense transcript, double-stranded RNA (dsRNA) would have been formed, which may theoretically trigger the interferon response [40, 41], leading to degradation of the dsRNA and thus disrupt mitochondrial gene expression. Therefore, the sense transcript, at least in theory, has to be processed as soon as being transcribed, and its corresponding antisense transcript must be prevented from annealing to the sense transcript. Initially, we predicted that mitosRNAs, which are mainly derived from the sense transcripts and thus mostly are in sense orientation, may represent an army of small RNAs that can target the antisense transcripts by annealing and inducing degradation of the antisense transcripts in a way similar to RNAi. Consequently, the antisense transcripts are prevented from interfering with the processing mechanisms of the sense transcripts (e.g., proper folding of tRNAs, translocation and translation of mRNAs, etc.). Our data from the in vitro transfection assays appeared to support such a hypothesis because overexpression of a sense-oriented mitosRNA increased levels of its host tRNA, whereas introduction of an antisense mitosRNA significantly reduced the levels of its host tRNA (Figure 4). If this hypothesis is correct, the lack of mitosRNAs would allow antisense transcripts to interfere with the processing of sense transcripts, and thus, once dsRNAs are formed, it may trigger the interferon response, leading to the degradation of double-stranded mitochondrial transcripts. However, we failed to detect dsRNA formation in Dicernull pachytene spermatocytes (data not shown), though levels of many mitosRNAs were much reduced, suggesting that this hypothesis may not hold true. Moreover, the fact that no known components of the RNAi machinery are present inside mitochondria suggests that mitosRNAs may not function as a post-transcriptional regulator by using the conventional RNAi mechanism. Therefore, mitochondria may possess a unique and independent RNAilike machinery, which allows post-transcriptional regulation by mitosRNAs. Alternatively, mitosRNAs may act as an epigenetic regulator affecting mitochondrial gene expression by modulating mitochondrial DNA methylation [42]. These intriguing hypotheses require further investigation in the future.

Our data also suggest that an unknown pathway exists within the mitochondria, which is responsible for the biogenesis of mitosRNAs. First, in both Dicer-null spermatocytes and Dicer-null Sertoli cells, not all of the mitosRNAs analyzed were abolished or significantly downregulated (Figure 7). Given the high cell purity (> $95 \%$ ), it is unlikely that those upregulated or largely unaffected mitosRNAs represented those carried over by contaminating cell types. A more plausible explanation is that, although Dicer inactivation can adversely affect mitosRNA production in the cell in general, a lack of DICER activity cannot completely abolish the production of mitosRNAs, suggesting that mitosRNAs are not the direct products of DICER. Second, the major size of mitosRNAs $(\sim 30 \mathrm{nt})$ is larger than that of DICER products, which is typically at $\sim 22 \mathrm{nt}$. The majority of mitosRNAs (90\%) range between 20-40 nt with a peak at 30 nt in size, and mitosRNAs belonging to the same clusters display overlapping sequences with 5 '-aligned, 3 '-aligned and staggered patterns, suggesting that these small RNAs are products of unknown ribonucleases present within the mitochondria. Third, no detectable levels of DICER 
or other components of the RNAi machinery were identified from the lysates of mitochondria purified from spermatocytes. This negative finding is consistent with the fact that datasets from two previous studies reporting that the whole proteome of mitochondria [37-39] contain neither DICER nor any of the known components of the RNAi machinery. Further investigation on the role of intramitochondrial ribonucleases may reveal the mechanism underlying the biogenesis of mitosRNAs. Given the lack of both DICER and RNAi machinery within mitochondria, it is likely that the altered mitosRNA production and dysregulated mitochondrial gene expression in the Dicer-deficient germ or somatic cells represent secondary effects of global defects in gene regulation due to DICER inactivation and miRNA/endo-siRNA deficiency. Nevertheless, DICER inactivation can affect not only the biogenesis of miRNAs and/or endo-siRNAs [17, 34], but also the production of other small RNA species, e.g., mitosRNAs. Thus, in addition to effects caused by the lack of miRNAs and/or endo-siRNAs, disrupted mitosRNA transcriptome may contribute to the phenotypes observed in numerous cell lineage-specific Dicer conditional knockout mouse lines [43-59].

In summary, data reported here demonstrate that the mammalian mitochondrial genome encodes numerous small noncoding RNAs, which may play an important role in mitochondrial and cellular functions. Dysregulation of mitosRNAs may be involved in human diseases and manipulation of these mitosRNAs may thus spell hopes for future therapeutics for such diseases.

\section{Materials and Methods}

\section{Small RNA isolation}

Small RNAs were isolated from mouse organs or cells using a mirVanaTM miRNA isolation kit according to the manufacturer's instructions (Ambion). Total RNAs from human brain, heart, stomach, small intestine and colon were purchased from BD Biosciences. Human small RNAs were then isolated from human total RNAs as described in the instructions of the miRNA isolation kit. The concentration of small RNAs was measured using a ND-1000 spectrophotometer (NanoDrop Technologies).

\section{Small noncoding RNA deep sequencing (sncRNA-Seq)}

For 454 sequencing, small RNA cDNA (srcDNA) libraries were prepared as described [60]. Briefly, small RNAs were polyadenylated and a 5'-RNA adapter was ligated with the poly(A)-tailed RNA. The ligation products were reverse-transcribed to make srcDNAs. srcDNAs from mouse small intestine and testis and human small intestine were amplified by PCR using two pairs of $\mathrm{PCR} /$ sequencing primers (long primers are F-A1 and F-B1r, short ones are Fus-A and Fus-B) (Supplementary information, Table S1) according to the instructions of the sequencing company (454 sequencing, now part of Roche). The PCR reaction was performed using $200 \mathrm{ng}$ of srcDNAs, $1 \mu \mathrm{l}$ of $10 \mathrm{pM}$ of the long primer pair,
$1 \mu \mathrm{l}$ of $2 \mathrm{pM}$ of the short primer pair, $0.5 \mu \mathrm{l}$ of $50 \times \mathrm{dNTP}, 5 \mu \mathrm{l}$ of $\mathrm{G} / \mathrm{C}$ melt, $1 \mu \mathrm{l}$ of $25 \mathrm{mM} \mathrm{Mg}(\mathrm{OAc}) 2,0.5 \mu \mathrm{l}$ of Advantage-GC 2 polymerase (Clontech) in step-down PCR conditions: $95^{\circ} \mathrm{C}$, $1 \mathrm{~min},\left(95^{\circ} \mathrm{C}, 30 \mathrm{~s} ; 62{ }^{\circ} \mathrm{C}, 30 \mathrm{~s} ; 68^{\circ} \mathrm{C}, 1 \mathrm{~min}\right) 2$ cycles, $\left(95^{\circ} \mathrm{C}\right.$, $\left.30 \mathrm{~s} ; 59{ }^{\circ} \mathrm{C}, 30 \mathrm{~s} ; 68{ }^{\circ} \mathrm{C}, 1 \mathrm{~min}\right) 3$ cycles, $\left(95^{\circ} \mathrm{C}, 30 \mathrm{~s} ; 56{ }^{\circ} \mathrm{C}, 30\right.$ $\left.\mathrm{s} ; 68^{\circ} \mathrm{C}, 1 \mathrm{~min}\right) 15$ cycles, and $68{ }^{\circ} \mathrm{C}, 5 \mathrm{~min}$. After PCR, $1 \mu \mathrm{l}$ of PCR products were analyzed on a $2 \%$ agarose gel. PCR products of $\sim 150-270$ bp were gel-eluted and purified using QIAquick Gel extraction kit (QIAGEN). The concentration of srcDNA libraries was measured. Three libraries (50 ng each) were sequenced with the sequencing primer Fus-A using the high-throughput Genome Sequencer 20 DNA Sequencing System (Roche).

For mitosRNA deep sequencing (mitosRNA-Seq) using the Ion Torrent PGM sequencer (Life Technologies), srcDNA libraries of WT or mtDNA-depleted (Rho0) PNT1A cells were prepared and sequenced using Ion 316 chips (Life Technologies) as described previously [34].

\section{Bioinformatic analysis}

Sequence reads were analyzed using our in-house sequence annotation software SeQuery v1.0 as described previously [61]. The sequences were first trimmed by removing the flanking linker and primer sequences. Mouse (NC_006914) and human (AC_000021) mitochondrial genome sequences were retrieved from the GenBank at the NCBI databases (http://www.ncbi.nlm.nih.gov/). By matching each of the sequencing reads to either the $\mathrm{H}$ or $\mathrm{L}$ strand of the mitochondrial genome, we extracted those that match perfectly to the mitochondrial genome and designated them as mitosRNAs. Identified mitosRNAs were then mapped onto mitochondrial genes. The number of mitosRNAs mapped to a particular mitochondrial gene and the size distribution of these mitosRNAs were analyzed and recorded. Sequence motifs of mitosRNAs were determined by analyzing the dominant occurrence of each of the four nucleotides (A, T, G, or C) at the first four positions at the 5'ends and the last 4 positions at the $3^{\prime}$-ends of mitosRNAs. Nucleotides with an occurrence rate of $>25 \%$ were plotted against their positions at either the $5^{\prime}$ or $3^{\prime}$ ends of mitosRNAs.

Deep sequencing reads were aligned to the mitochondrial genome (hg19) with tMAP (Life Technologies) and subsequently matched to annotated mitosRNA sequences with Sequery v1.0. Raw reads were normalized to account for sequencing depth. This was achieved by tMAP alignment to hg19 (not including the mitochondrial genome), calculating the ratio difference in reads between Rho0 (mtDNA-depleted) and WT PNT1A cell libraries ( 0.492), and multiplying the WT reads by this ratio to adjust for differences in sequencing depth. Normalized values were used to calculate expression values relative to PNT1A WT expression where all normalized values were divided by the corresponding wild-type normalized value. Heat map showing the relative expression for each of the mitosRNAs in WT or Rho0 (mtDNAdepleted) PNT1A cells was generated using the Partek Genomics Suite 6.6 (Partek).

\section{MitosRA nomenclature}

MitosRNA sequences were named based on the mitochondrial genes (37 in total) or two mitochondrial genomic regions including the origin of replication for L strand (OL) region and the D-loop region, from which mitosRNAs are derived from or are complementary to. All individual mitosRNAs (1 499 in mice and 2540 in 
humans) were clustered into a total of 39 clusters of mitosRNAs (Supplementary information, Tables S2-S5). Most of the mitosRNAs were mapped to one gene, but if a sequence spanned more than one mitochondrial gene/region, the direction of the overrun segment was indicated after the abbreviated gene name by either "+", which stands for a overrun extending toward upstream in a $3^{\prime} \rightarrow 5^{\prime}$ direction, or " -", which means a overrun towards the downstream in a $5^{\prime} \rightarrow 3^{\prime}$ direction. Antisense sequences were indicated by an asterisk ("*”) after the abbreviated gene name or overrun indicator (if present). Multiple sequences matching to a particular gene were generally ordered so that sequences were numbered from their start position closest to the start of the gene. A mitosRNA name follows the format: [species]-mitosR-[H or L strand][mitochondrial gene/genomic region] [overrun direction "+” or “- "][antisense “*”]_[number], e.g., 'hsa-mitosR-H-Q*_1-5 indicates a human mitosRNA cluster containing 5 individual mitosRNAs (1-5) that are derived from the H-strand gene TrnQ in an antisense orientation; "mmu-mitosR-H-R1-1-87" refers to a murine mitosRNA cluster consisting of 87 individual mitosRNAs derived from the H-strand gene Rnrl in a sense orientation. "hsa-mitosR$\mathrm{L}-\mathrm{Y}+$ 3" indicates a human mitosRNA derived from the L-strand gene $\overline{T r n Y}$, but a small portion was matched to an upstream gene in the $3^{\prime} \rightarrow 5^{\prime}$ direction ( $\operatorname{TrnC}$ ).

\section{PCR-based quantification of mitosRNA}

Preparation of the srcDNAs and PCR analyses of mitosRNAs were performed as described [28]. Primer sequences used for qPCR-based expression profiling are shown in Supplementary information, Table S1. TaqMan-based real-time PCR was used in small RNA quantification and U6 snRNA was used as the endogenous control, as described previously [17]. Large RNA cDNAs were used as PCR templates to quantify mitochondria rRNAs and mRNAs. SYBR Green-based real-time PCR was used in large RNA quantification and Gapdh mRNA was used as an endogenous control as described [27, 60, 62].

\section{Confirmation of the 5' phosphate in mitosRNAs}

Small RNAs isolated from mouse and human small intestines were polyadenylated, and poly(A)-tailed small RNAs were then purified as described [28]. Purified small RNAs $(\sim 1 \mu \mathrm{g})$ were treated with or without $2 \mu \mathrm{l}(2 \mathrm{U})$ of calf intestinal alkaline phosphatase (CIAP) (Promega) in a reaction volume of $50 \mu \mathrm{l}$, and the reaction mixtures were incubated at $37^{\circ} \mathrm{C}$ for $1 \mathrm{~h}$. RNA samples were purified by phenol:chloroform and column extraction [28]. About $2 \mu \mathrm{g}$ of poly(A)-tailed and dephosphorylated RNAs were ligated with 3 $\mu \mathrm{g}$ of a 5' RNA adapter, and ligated RNAs were purified again [60]. Ligated RNAs were reverse-transcribed with the RTQ primer [28, 60]. srcDNAs were amplified with a long $5^{\prime}$ linker primer miRT2, followed with a short primer miP2 by PCR (Supplementary information, Table S1). The PCR reaction was performed in a $25 \mu 1$ reaction volume containing $12.5 \mu \mathrm{l}$ of $2 \times$ GoTaq Green Master Mix (Promega), $5 \mu \mathrm{l}$ of the synthesized srcDNA, $10 \mathrm{mM}$ of miRT-2, and $10 \mathrm{mM}$ of RTQ using the GeneAmp PCR system 2700 (Applied Biosystems). Stepdown PCR conditions were as follows: $95{ }^{\circ} \mathrm{C}$, $2 \mathrm{~min},\left(95^{\circ} \mathrm{C}, 1 \mathrm{~min} ; 63{ }^{\circ} \mathrm{C}, 30 \mathrm{~s} ; 72^{\circ} \mathrm{C}, 1 \mathrm{~min}\right) 2$ cycles, $\left(95^{\circ} \mathrm{C}\right.$, $\left.1 \mathrm{~min} ; 60^{\circ} \mathrm{C}, 30 \mathrm{~s} ; 72^{\circ} \mathrm{C}, 1 \mathrm{~min}\right) 2$ cycles and $\left(95^{\circ} \mathrm{C}, 1 \mathrm{~min} ; 57\right.$ $\left.{ }^{\circ} \mathrm{C}, 30 \mathrm{~s} ; 72{ }^{\circ} \mathrm{C}, 1 \mathrm{~min}\right) 3$ cycles. After the PCR cycles, $10 \mathrm{mM}$ of miP2 was added to the reaction and returned for additional cycles on the PCR system. Additional cycles were: $95^{\circ} \mathrm{C}, 2 \mathrm{~min},\left(95^{\circ} \mathrm{C}\right.$, $\left.1 \mathrm{~min} ; 60{ }^{\circ} \mathrm{C}, 30 \mathrm{~s} ; 72^{\circ} \mathrm{C}, 1 \mathrm{~min}\right) 10$ cycles, $\left(95^{\circ} \mathrm{C}, 1 \mathrm{~min} ; 57^{\circ} \mathrm{C}\right.$, $\left.30 \mathrm{~s} ; 72{ }^{\circ} \mathrm{C}, 1 \mathrm{~min}\right) 20$ cycles and $72{ }^{\circ} \mathrm{C}, 5 \mathrm{~min}$. After PCR, $75 \mu \mathrm{l}$ of nuclease-free water was added to the srcDNAs. Each mitosRNA was amplified with a mitosRNA-specific reverse primer and the universal reverse primer RT-UN1r by PCR. The PCR reaction was performed as described above except for using $1.5 \mu \mathrm{l}$ of the amplified srcDNA and $10 \mathrm{mM}$ of each mitosRNA-specific reverse primer (Supplementary information, Table S1). The PCR conditions were: $95^{\circ} \mathrm{C}, 2 \mathrm{~min},\left(95^{\circ} \mathrm{C}, 1 \mathrm{~min} ; 55^{\circ} \mathrm{C}, 30 \mathrm{~s} ; 72^{\circ} \mathrm{C}, 1 \mathrm{~min}\right)$ 30 cycles, and $72{ }^{\circ} \mathrm{C}, 5 \mathrm{~min}$. After PCR, $2 \mu 1$ of the PCR products were analyzed on a $2 \%$ agarose gel. All PCR products were gelextracted and subcloned into pcDNA3.1 TOPO cloning vector [60]. The cloned PCR products were amplified by colony-direct PCR, purified and sequenced with primers pcDNA and BGHr as described [60] at the Nevada Genomics Center (University of Nevada, Reno, USA).

\section{Mitochondria purification}

For northern blot analyses, mitochondrial and cytosolic fractions were isolated from brain or liver using a Mitochondria Isolation Kit (Pierce) according to the manufacturer's protocol. Briefly, $\sim 200 \mathrm{mg}$ of brain or liver tissue was washed twice with $1.5 \mathrm{ml}$ PBS and cut into small pieces followed by addition of $800 \mu \mathrm{l}$ of PBS. Tissue was disrupted using a tissue homogenizer TissueTearor (BioSpec) on ice followed by centrifugation at $1000 \times g$ for $3 \mathrm{~min}$ at $4{ }^{\circ} \mathrm{C}$ and then the supernatant was discarded. The pellet was resuspended in $800 \mu \mathrm{l}$ of BSA/Reagent A Solution, vortexed at medium speed for $5 \mathrm{~s}$, and incubated on ice for $2 \mathrm{~min}$. After incubation, $10 \mu \mathrm{l}$ of Mitochondria Isolation Reagent B was added, and the tube was vortexed at maximum speed for $5 \mathrm{~s}$. The tube was incubated on ice for $5 \mathrm{~s}$, vortexing at maximum speed every minute. After incubation, $800 \mu 1$ of Mitochondria Isolation Reagent $\mathrm{C}$ was added and mixed. The tube was centrifuged at $700 \times \mathrm{g}$ for $10 \mathrm{~min}$ at $4{ }^{\circ} \mathrm{C}$. The supernatant was transferred to a new tube whereas the pellet containing the nucleus and cell debris was saved for small RNA isolation. Supernatant was centrifuged at $3000 \times g$ for $15 \mathrm{~min}$ at $4{ }^{\circ} \mathrm{C}$. The supernatant was removed from the mitochondrial pellet. The supernatant (cytosolic fraction) was also saved for small RNA isolation. The mitochondrial pellet was washed with $500 \mu \mathrm{l}$ of Wash Buffer, centrifuged at $12000 \times g$ for 5 min at $4{ }^{\circ} \mathrm{C}$ and the supernatant was discarded. Five mitochondrial pellets were combined and used to isolate small RNAs along with the two saved fractions (nucleus and cytosolic fractions).

For western blot analyses, mitochondria were isolated from human HEK293T cells using magnetic beads-based mitochondria isolation Kit (Miltenyi Biotec) according to the manufacturer's instructions. Briefly, anti-TOMM22 magnetic beads were added to the cell lysates to label the mitochondria, followed by mitochondria isolation in the magnetic field. Isolated mitochondria and total cell lysates were collected for western blot analyses.

\section{Small RNA northern blot analysis}

For mitosRNA detection, northern blot analyses were performed using a mirVana miRNA Probe Construction Kit (Ambion). For transcription of antisense RNA probes, DNA oligonucleotides complementary to mitosR-L-A_3, mitosR-H-A*_1, mitosR-L$\mathrm{P}+\_6$, and mitosR-H-P*__ 3 with the $12 \mathrm{nt}$ linker sequence were synthesized in IDT, Inc. (Supplementary information, Table S1). A 12 -nt sequence is complementary to the $\mathrm{T} 7$ promoter primer 
provided in the Kit. Each synthesized oligonucleotide $(200 \mu \mathrm{M})$ was annealed with $2 \mu \mathrm{l}$ of the T7 promoter primer in $6 \mu \mathrm{l}$ of DNA hybridization buffer at $70{ }^{\circ} \mathrm{C}$ for $5 \mathrm{~min}$, followed by incubation at room temperature (RT) for $5 \mathrm{~min}$. To prepare for dsDNA template, end-filling reaction was performed by adding $2 \mu 1$ of Klenow Reaction Buffer, $2 \mu 1$ of $10 \times$ dNTP Mix, $4 \mu$ of nuclease-free water and $2 \mu \mathrm{l}$ of Exo-Klenow at $37{ }^{\circ} \mathrm{C}$ for $30 \mathrm{~min}$. In vitro transcription was carried out to make RNA probes in a $20-\mu 1$ reaction containing $1 \mu \mathrm{l}$ dsDNA template, $2 \mu 110 \times$ Transcription buffer, 1 $\mu 110 \mathrm{mM}$ ATP, $1 \mu 110 \mathrm{mM}$ GTP, $1 \mu 110 \mathrm{mM} \mathrm{CTP}, 5 \mu 1\left[\alpha{ }^{-32} \mathrm{P}\right]$ $\mathrm{UTP}$ at $800 \mathrm{Ci} / \mathrm{mmol}$ and $10 \mathrm{mCi} / \mathrm{ml}(3.125 \mu \mathrm{M})$ and $2 \mu \mathrm{T} 7$ RNA Polymerase at $37^{\circ} \mathrm{C}$ for $15 \mathrm{~min}$ as described in the mirVana miRNA Probe Construction Kit protocol. After reaction, DNase I was added and incubated at $37{ }^{\circ} \mathrm{C}$ for $10 \mathrm{~min}$. The labeled RNA probes were purified using a purification filter cartridge provided in the mirVana Probe \& Marker Kit (Ambion). Briefly, the Binding/Washing Buffer (12 volumes of the sample) was added to the sample and mixed thoroughly. The mixture was applied to a purification filter cartridge and centrifuged. The cartridge was washed with $300 \mu \mathrm{l}$ of the Binding/Washing Buffer. RNA probes were recovered with two sequential elutions using $20 \mu \mathrm{l}$ of preheated (95 ${ }^{\circ} \mathrm{C}$ ) Elution Buffer each time. The yield of the radiolabeled RNA probes was determined by scintillation counting using Ecoscint $\mathrm{O}$ (National Diagnostics). About $2.5 \mu \mathrm{g}$ of small RNAs isolated from the brain, heart, liver, small intestine, testis, ovary, liver or brain subcellular fractions (mitochondrial, cytoplasmic and nucleus) were used for northern blotting. To remove DNA from the RNA samples, DNA removal reaction was carried out using DNA-free Kit (Ambion) according to the manufacturer's protocol except for adding $1 \mu$ of Recombinant Ribonuclease Inhibitor (Ambion) to each sample to protect the RNAs from RNases. The RNA samples were recovered by centrifugation at $9000 \mathrm{rpm}$ for $5 \mathrm{~min}$. Concentration of the recovered RNAs was measured using the ND-1000 spectrophotometer (NanoDrop Technologies, Inc.). About $2 \mu \mathrm{g}$ of the RNAs were mixed with equal amounts of Gel Loading Buffer II provided in the mirVana Probe \& Marker Kit. The samples were incubated at $95{ }^{\circ} \mathrm{C}$ for $3 \mathrm{~min}$ and separated on a $15 \%$ denaturing polyacrylamide gel. RNAs were transferred onto a blot membrane Hybond-XL (Amersham Biosciences) using the Panther Semidry Electroblotter (Owl Separation Systems). Prehybridization of blots in ULTRAhyb-Oligo Hybridization Buffer (Ambion) was carried out at $65{ }^{\circ} \mathrm{C}$ for $30 \mathrm{~min}$ followed by $42{ }^{\circ} \mathrm{C}$ for $30 \mathrm{~min}$. About $1 \mu \mathrm{l}$ $(\sim 1 \times 107 \mathrm{cpm})$ of the purified RNA probe was diluted into a total volume of $10 \mu$ with TE buffer, denatured at $93{ }^{\circ} \mathrm{C}$ for $2 \mathrm{~min}$ and added to the prehybridization solution. The blot was hybridized at $42{ }^{\circ} \mathrm{C}$ for $12 \mathrm{~h}$. After hybridization, the blot was washed in NorthernMax Low Stringency Wash Buffer (Ambion) three times for 5 min at RT followed by $30 \mathrm{~min}$ at $42{ }^{\circ} \mathrm{C}$. The blot was wrapped in a plastic wrap, exposed to a phosphorimager, and scanned on the ChemiDoc XRS and Molecular Imager FX imaging system (BioRad). The blot was partially stripped and hybridized with the $5 \mathrm{~S}$ rRNA probe labeled with the same $\left[\alpha-{ }^{32} \mathrm{P}\right]$ UTP for a RNA loading control. 5S rRNA was used as an endogenous loading control (Figure 4). Although it is encoded in the nuclear genome, high levels of 5S rRNA have been detected in mammalian mitochondria [63$66]$.

\section{Small RNA transfection assays}

Antisense and sense oligo RNAs (Supplementary information,
Table S1) were synthesized in a 100 -nmol scale with a standard desalting method (IDT) or in a 250-nmol scale with HPLC purification (Exiqon). Synthesized RNAs were diluted in $5 \times$ siRNA buffer (Dharmacon) in a concentration of $5 \mu \mathrm{g} / \mu \mathrm{l}$, and diluted RNAs were incubated at $90{ }^{\circ} \mathrm{C}$ for $1 \mathrm{~min}$ and $50{ }^{\circ} \mathrm{C}$ for $10 \mathrm{~min}$. Antisense or sense RNA oligos were transfected into NIH $3 \mathrm{~T} 3$ cells grown on a 6-well plate using the RNAiFect transfection reagent (QIAGEN). $1 \mu \mathrm{l}$ of RNA oligos $(5 \mu \mathrm{g})$ was combined with $8 \mu$ of RNAiFect in $100 \mu \mathrm{l}$ of EC-R buffer. The RNA reaction was incubated at RT for 10 min and RNA complexes were added to 3 T3 cells. The cells were rocked gently in a $\mathrm{CO} 2$ incubator at $37^{\circ} \mathrm{C}$ for $30 \mathrm{~min}$. The transfected $3 \mathrm{~T} 3$ cells were cultured for $24 \mathrm{~h}$. The cells were washed with PBS buffer and trypsinized with $0.25 \%$ trypsin (Invitrogen). Small RNAs were isolated from the cells. SrcDNAs were prepared and qPCR was performed and analyzed as described [28].

\section{Preparation of mtDNA-depleted PNT1A cells}

mtDNA-depleted PNT1A cells were prepared as described previously [33]. Briefly, PNT1A cells were grown in RPMI-1640 supplemented with $10 \%$ heat-inactivated fetal bovine serum (FBS). PNT1A cells were treated with ethidium bromide (EtBr, $100 \mathrm{ng}$ / $\mathrm{ml}$ ) to induce mtDNA depletion. After 30 days of culture in the presence of EtBr, cells were screened for mtDNA content by PCR and Southern hybridization. To generate mtDNA-less (Rho0) PNT1A cells, mtDNA-depleted cells were grown for additional 15 days in the presence of EtBr. The complete depletion of mtDNA was confirmed as described previously [33].

Purification of Dicer-null or Drosha-null pachytene spermatocytes and Dicer-null testicular Sertoli cells

Pachytene spermatocytes were purified from the testes of male germline-specific Dicer cKO (Dicer ${ }^{\text {lox/lox }}$, Rosa26mTmG+/ tg, Stra8-iCre) and control (Rosa26mTmG+/tg, Stra8-iCre) mice using the STA-PUT method described previously [17, 34, 67]. Sertoli cells were purified from the testes of a Sertoli cell-specific Dicer cKO (Dicerlox/lox, Rosa26mTmG+/tg, Amh-Cre) and control (Rosa26mTmG+/tg, Amh-Cre) mice [35] using fluorescenceactivated cell sorting (FACS) on a Becton Dickinson FACSAria II. The generation and characterization of these conditional knockout mouse lines were described previously [17, 34, 35].

\section{Western blot analyses}

Protein isolation and western blot analyses were performed as described [68-70]. GAPDH was used as a loading control. The mouse anti-MTCO1 (Cat\# ab14705), the mouse anti-COX4 (Cat\# ab14744) and the mouse anti-TOMM22 (Cat\# ab57523) monoclonal antibodies were purchased from Abcam Inc. Rabbit antiGAPDH (Cell Signaling Technology, Cat\# T4026) polyclonal antibodies were used. The rabbit anti-DICER and rabbit anti-AGO2 polyclonal antibodies were generated by GenScript Inc. according to our antigen design and the specificity of these antibodies has been verified previously $[17,34]$.

\section{Acknowledgments}

We would like to thank R Keegan Idler for critical reading of the manuscript. This study was supported by the National Institutes of Health (NIH) (HD050281, HD060858 and HD071736 to WY) and United States Army (W81XWH-10-1-0176 to J-TH). 
The Sequery v1.0 software for genome sequence annotation analyses was developed by the Imaging Core (Core D), and breeding and genotyping of all of the three conditional knockout mouse lines and FACS were performed in the Molecular and Transgenics Core (Core B), with supports by Centers of Biomedical Research Excellence Grant P20-RR18751 from the NIH/NCRR.

\section{References}

1 Taylor RW, Turnbull DM. Mitochondrial DNA transcription: regulating the power supply. Cell 2007; 130:211-213.

2 Scarpulla RC. Transcriptional paradigms in mammalian mitochondrial biogenesis and function. Physiol Rev 2008; 88:611638.

3 Guarente L. Mitochondria - a nexus for aging, calorie restriction, and sirtuins? Cell 2008; 132:171-176.

4 Chan DC. Mitochondria: dynamic organelles in disease, aging, and development. Cell 2006; 125:1241-1252.

5 Balaban RS, Nemoto S, Finkel T. Mitochondria, oxidants, and aging. Cell 2005; 120:483-495.

6 Newmeyer DD, Ferguson-Miller S. Mitochondria: releasing power for life and unleashing the machineries of death. Cell 2003; 112:481-490.

7 Fernandez-Silva P, Enriquez JA, Montoya J. Replication and transcription of mammalian mitochondrial DNA. Exp Physiol 2003; 88:41-56.

8 Gaspari M, Larsson NG, Gustafsson CM. The transcription machinery in mammalian mitochondria. Biochim Biophys Acta 2004; 1659:148-152.

9 Montoya J, Lopez-Perez MJ, Ruiz-Pesini E. Mitochondrial DNA transcription and diseases: past, present and future. Biochim Biophys Acta 2006; 1757:1179-1189.

10 Wallace DC. Mitochondrial diseases in man and mouse. Science 1999; 283:1482-1488.

11 Lagos-Quintana M, Rauhut R, Yalcin A, Meyer J, Lendeckel W, Tuschl T. Identification of tissue-specific microRNAs from mouse. Curr Biol 2002; 12:735-739.

12 Jones L. Revealing micro-RNAs in plants. Trends Plant Sci 2002; 7:473-475.

13 Lim LP, Lau NC, Weinstein EG, et al. The microRNAs of Caenorhabditis elegans. Genes Dev 2003; 17:991-1008.

14 Aravin A, Gaidatzis D, Pfeffer S, et al. A novel class of small RNAs bind to MILI protein in mouse testes. Nature 2006; 442:203-207.

15 Girard A, Sachidanandam R, Hannon GJ, Carmell MA. A germline-specific class of small RNAs binds mammalian Piwi proteins. Nature 2006; 442:199-202.

16 Lau NC, Seto AG, Kim J, et al. Characterization of the piRNA complex from rat testes. Science 2006; 313:363-367.

17 Song R, Hennig GW, Wu Q, Jose C, Zheng H, Yan W. Male germ cells express abundant endogenous siRNAs. Proc Natl Acad Sci USA 2011; 108:13159-13164.

18 Suh N, Blelloch R. Small RNAs in early mammalian development: from gametes to gastrulation. Development 2011; 138:1653-1661.

19 Suh N, Baehner L, Moltzahn F, et al. MicroRNA function is globally suppressed in mouse oocytes and early embryos. Current Biol 2010; 20:271-277.

20 Babiarz JE, Ruby JG, Wang Y, Bartel DP, Blelloch R. Mouse
ES cells express endogenous shRNAs, siRNAs, and other Microprocessor-independent, Dicer-dependent small RNAs. Genes Dev 2008; 22:2773-2785.

21 Taft RJ, Kaplan CD, Simons C, Mattick JS. Evolution, biogenesis and function of promoter-associated RNAs. Cell Cycle 2009; 8:2332-2338.

22 Napoli S, Pastori C, Magistri M, Carbone GM, Catapano CV. Promoter-specific transcriptional interference and $c-m y c$ gene silencing by siRNAs in human cells. EMBO J 2009; 28:17081719.

23 Hawkins PG, Santoso S, Adams C, Anest V, Morris KV. Promoter targeted small RNAs induce long-term transcriptional gene silencing in human cells. Nucleic Acids Res 2009; 37:2984-2995.

24 Kim VN, Han J, Siomi MC. Biogenesis of small RNAs in animals. Nat Rev Mol Cell Biol 2009; 10:126-139.

25 Han J, Pedersen JS, Kwon SC, et al. Posttranscriptional crossregulation between Drosha and DGCR8. Cell 2009; 136:7584.

26 Babiarz JE, Blelloch R. Small RNAs - their biogenesis, regulation and function in embryonic stem cells. StemBook. Cambridge (MA) 2008.

27 Ro S, Song R, Park C, Zheng H, Sanders KM, Yan W. Cloning and expression profiling of small RNAs expressed in the mouse ovary. RNA 2007; 13:2366-2380.

28 Ro S, Park C, Jin J, Sanders KM, Yan W. A PCR-based method for detection and quantification of small RNAs. Biochem Biophys Res Commun 2006; 351:756-763.

29 Zamore PD, Tuschl T, Sharp PA, Bartel DP. RNAi: doublestranded RNA directs the ATP-dependent cleavage of mRNA at 21 to 23 nucleotide intervals. Cell 2000; 101:25-33.

30 Perna NT, Kocher TD. Mitochondrial DNA: molecular fossils in the nucleus. Current Biol 1996; 6:128-129.

31 Yao YG, Kong QP, Salas A, Bandelt HJ. Pseudomitochondrial genome haunts disease studies. J Med Genet 2008; 45:769772.

32 Yuan JD, Shi JX, Meng GX, An LG, Hu GX. Nuclear pseudogenes of mitochondrial DNA as a variable part of the human genome. Cell Res 1999; 9:281-290.

33 Moro L, Arbini AA, Yao JL, di Sant'Agnese PA, Marra E, Greco M. Mitochondrial DNA depletion in prostate epithelial cells promotes anoikis resistance and invasion through activation of PI3K/Akt2. Cell Death Differ 2009; 16:571-583.

$34 \mathrm{Wu} \mathrm{Q}$, Song R, Ortogero N, et al. The RNase III enzyme DROSHA is essential for microRNA production and spermatogenesis. J Biol Chem 2012; 287:25173-25190.

35 Papaioannou MD, Pitetti JL, Ro S, et al. Sertoli cell Dicer is essential for spermatogenesis in mice. Dev Biol 2009; 326:250-259.

36 Agrawal N, Dasaradhi PV, Mohmmed A, Malhotra P, Bhatnagar RK, Mukherjee SK. RNA interference: biology, mechanism, and applications. Microbiol Mol Biol Rev 2003; 67:657685.

37 Taylor SW, Fahy E, Zhang B, et al. Characterization of the human heart mitochondrial proteome. Nat Biotechnol 2003; 21:281-286.

38 Da Cruz S, Xenarios I, Langridge J, Vilbois F, Parone PA, Martinou JC. Proteomic analysis of the mouse liver mitochondrial inner membrane. J Biol Chem 2003; 278:41566-41571. 
39 Zhang J, Li X, Mueller M, et al. Systematic characterization of the murine mitochondrial proteome using functionally validated cardiac mitochondria. Proteomics 2008; 8:1564-1575.

40 Chakrabarti A, Jha BK, Silverman RH. New insights into the role of RNase L in innate immunity. J Interferon Cytokine Res 2011; 31:49-57.

41 Barber GN. Host defense, viruses and apoptosis. Cell Death Differ 2001; 8:113-126.

42 Shock LS, Thakkar PV, Peterson EJ, Moran RG, Taylor SM. DNA methyltransferase 1, cytosine methylation, and cytosine hydroxymethylation in mammalian mitochondria. Proc Natl Acad Sci USA 2011; 108:3630-3635.

43 Babiarz JE, Hsu R, Melton C, Thomas M, Ullian EM, Blelloch R. A role for noncanonical microRNAs in the mammalian brain revealed by phenotypic differences in Dgcr8 versus Dicer1 knockouts and small RNA sequencing. RNA 2011; 17:1489-1501.

44 Bezman NA, Cedars E, Steiner DF, Blelloch R, Hesslein DG, Lanier LL. Distinct requirements of microRNAs in NK cell activation, survival, and function. J Immunol 2010; 185:38353846.

45 Damiani D, Alexander JJ, O'Rourke JR, et al. Dicer inactivation leads to progressive functional and structural degeneration of the mouse retina. J Neurosci 2008; 28:4878-4887.

46 Georgi SA, Reh TA. Dicer is required for the transition from early to late progenitor state in the developing mouse retina. $J$ Neurosci 2010; 30:4048-4061.

47 Harvey SJ, Jarad G, Cunningham J, et al. Podocyte-specific deletion of dicer alters cytoskeletal dynamics and causes glomerular disease. J Am Soc Nephrol 2008; 19:2150-2158.

48 Hayashi K, Chuva de Sousa Lopes SM, Kaneda M, et al. MicroRNA biogenesis is required for mouse primordial germ cell development and spermatogenesis. PLoS One 2008; 3:e1738.

49 Huang T, Liu Y, Huang M, Zhao X, Cheng L. Wnt1-cremediated conditional loss of Dicer results in malformation of the midbrain and cerebellum and failure of neural crest and dopaminergic differentiation in mice. J Mol Cell Biol 2010; 2:152-163.

50 Iida A, Shinoe T, Baba Y, Mano H, Watanabe S. Dicer plays essential roles for retinal development by regulation of survival and differentiation. Invest Ophthalmol Vis Sci 2011; 52:3008-3017.

51 Kim GJ, Georg I, Scherthan H, et al. Dicer is required for Sertoli cell function and survival. Int J Dev Biol 2010; 54:867875.

52 Lei L, Jin S, Gonzalez G, Behringer RR, Woodruff TK. The regulatory role of Dicer in folliculogenesis in mice. Mol Cell Endocrinol 2010; 315:63-73.

53 Mudhasani R, Puri V, Hoover K, Czech MP, Imbalzano AN, Jones SN. Dicer is required for the formation of white but not brown adipose tissue. J Cell Physiol 2011; 226:1399-1406.

54 Nagaraja AK, Andreu-Vieyra C, Franco HL, et al. Deletion of Dicer in somatic cells of the female reproductive tract causes sterility. Mol Endocrinol 2008; 22:2336-2352.

55 Pan Y, Balazs L, Tigyi G, Yue J. Conditional deletion of Dicer in vascular smooth muscle cells leads to the developmental delay and embryonic mortality. Biochem Biophys Res Commun 2011; 408:369-374.

56 Sekine S, Ogawa R, Ito R, et al. Disruption of Dicer1 induces dysregulated fetal gene expression and promotes hepatocarcinogenesis. Gastroenterology 2009; 136:2304-2315. e1-4.

57 Sequeira-Lopez ML, Weatherford ET, Borges GR, et al. The microRNA-processing enzyme dicer maintains juxtaglomerular cells. J Am Soc Nephrol 2010; 21:460-467.

58 Zhao J, Lee MC, Momin A, et al. Small RNAs control sodium channel expression, nociceptor excitability, and pain thresholds. J Neurosci 2010; 30:10860-10871.

59 Zhou X, Jeker LT, Fife BT, et al. Selective miRNA disruption in T reg cells leads to uncontrolled autoimmunity. J Exp Med 2008; 205:1983-1991.

60 Ro S, Park C, Song R, et al. Cloning and expression profiling of testis-expressed piRNA-like RNAs. RNA 2007; 13:16931702.

61 Ortogero N, Hennig GW, Langille C, Ro S, McCarrey JR, Yan W. Computer-Assisted Annotation of Murine Sertoli Cell Small RNA Transcriptome. Biol Reprod 2013; 3;88:3

62 Ro S, Park C, Young D, Sanders KM, Yan W. Tissue-dependent paired expression of miRNAs. Nucleic Acids Res 2007; 35:5944-5953.

63 Entelis NS, Kolesnikova OA, Dogan S, Martin RP, Tarassov IA. 5S rRNA and tRNA import into human mitochondria. Comparison of in vitro requirements. J Biol Chem 2001; 276:45642-45653.

64 Entelis NS, Kolesnikova OA, Martin RP, Tarassov IA. RNA delivery into mitochondria. Adv Drug Deliv Rev 2001; 49:199-215.

65 Magalhaes PJ, Andreu AL, Schon EA. Evidence for the presence of 5S rRNA in mammalian mitochondria. Mol Biol Cell 1998; 9:2375-2382.

66 Yoshionari S, Koike T, Yokogawa T, et al. Existence of nuclear-encoded 5S-rRNA in bovine mitochondria. FEBS Lett 1994; 338:137-142.

67 Song R, Ro S, Michaels JD, Park C, McCarrey JR, Yan W. Many X-linked microRNAs escape meiotic sex chromosome inactivation. Nat Genet 2009; 41:488-493.

68 Zheng H, Stratton CJ, Morozumi K, Jin J, Yanagimachi R, Yan W. Lack of Spem1 causes aberrant cytoplasm removal, sperm deformation, and male infertility. Proc Natl Acad Sci USA 2007; 104:6852-6857.

69 Yan W, Ma L, Burns KH, Matzuk MM. Haploinsufficiency of kelch-like protein homolog 10 causes infertility in male mice. Proc Natl Acad Sci USA 2004; 101:7793-7798.

70 Yan W, Ma L, Burns KH, Matzuk MM. HILS1 is a spermatidspecific linker histone H1-like protein implicated in chromatin remodeling during mammalian spermiogenesis. Proc Natl Acad Sci USA 2003; 100:10546-10551.

(Supplementary information is linked to the online version of the paper on the Cell Research website.) 